\title{
A spatial study of the mid-IR emission features in four Herbig Ae/Be stars
}

\author{
C. Boersma ${ }^{1}$, E. Peeters ${ }^{2,3,4}$, N. L. Martín-Hernández ${ }^{5}$, G. van der Wolk $^{1}$, A. P. Verhoeff ${ }^{6}$, A. G. G. M. Tielens ${ }^{7}$, \\ L. B. F. M. Waters ${ }^{6}$, and J. W. Pel ${ }^{1}$ \\ 1 Kapteyn Astronomical Institute, PO Box 800, 9700 AV, Groningen, The Netherlands \\ e-mail: boersma@astro.rug.nl \\ 2 Department of Physics and Astronomy, University of Western Ontario, London, ON N6A 3K7, Canada \\ SETI Institute, 515 N. Whisman Road, Mountain View, CA 94043, USA \\ 4 NASA-Ames Research Center, Mail Stop 245-6, Moffet Field, CA 94035, USA \\ 5 Instituto de Astrofísica de Canarias, vía Láctea s/n, E38205 La Laguna, Spain \\ 6 Astronomical Institute "Anton Pannekoek", University of Amsterdam, Kruislaan 403, 1098 SJ Amsterdam, The Netherlands \\ 7 NASA-Ames Research Center, Mail Stop 245-3, Moffet Field, CA 94035, USA
}

Received 10 February 2009 / Accepted 9 May 2009

ABSTRACT

\begin{abstract}
Context. Infrared (IR) spectroscopy and imaging provide a prime tool to study the characteristics of polycyclic aromatic hydrocarbon (PAH) molecules and the mineralogy in regions of star formation. Herbig Ae/Be stars are known to have varying amounts of natal cloud material present in their vicinity.

Aims. Our aim is to study the characteristics of the mid-IR emission originating in Herbig Ae/Be stars, especially the extent of the emission and how this relates to the (proto-)stellar characteristics.

Methods. Today's powerful ground- and space-based telescopes provide images and spectra at unprecedented spectral and spatial resolution. We analyse the images and spectra from four Herbig Ae/Be stars (IRAS 06084-0611, CD-42 11721, TY CrA, and HD 176386), as obtained with TIMMI2 on the ESO $3.6 \mathrm{~m}$ telescope and VISIR on the VLT. These observations are supplemented with data from ISO-SWS and Spitzer-IRAC.

Results. We find evidence for large-scale structure and extended emission in all four sources, except for HD 176386, which only shows silicate emission, all sources show PAH emission in their spectra. In addition, a spatially resolved silicate and PAH spectrum could be extracted for TY CrA.

Conclusions. The variety in emission scales distinguishes two classes. In the first, the morphology and spectral characteristics resemble those of reflection nebulae. In the second, the characteristics are in-line with Herbig A stars. This separation simply reflects a difference in stellar characteristics (e.g. luminosity). In Herbig B stars, dust emission from the surroundings dominates, where for Herbig A stars, the disk dominates the emission. In this scheme, IRAS 06084-0611 and CD-42 11721 resemble reflection nebulae and HD 176386 a more typical Herbig Ae/Be star. TY CrA shows characteristics common to both genuine reflection nebulae and Herbig B stars. We propose a geometry for TY CrA, with most notably, a 70 AU inner gap in the 340 AU circumtertiary disk cleared by a fourth stellar companion.
\end{abstract}

Key words. techniques: spectroscopic - infrared: ISM - ISM: molecules - astrochemistry

\section{Introduction}

Herbig Ae/Be stars are pre-main sequence stars of intermediate mass and spectral class A or B that exhibit emission lines and have associated nebulosities. Many Herbig Ae/Be stars show an infrared excess of thermal origin and non-zero polarisation at optical wavelengths. These are signs for circumstellar material, possibly distributed in a proto-planetary disk (Herbig 1960). Often, Herbig Ae/Be stars are considered as the younger/heavier counterparts of T Tauri stars (Waters \& Waelkens 1998).

Herbig Ae/Be stars form in large molecular clouds, which initially can obscure them from sight. When the stellar light is able to break through, the star starts to illuminate its surroundings. Continuing their pre-main sequence evolution, the star will evacuate a cavity in the surrounding medium (e.g. NGC 2023 and NGC 7023). The (proto-)stellar mass determines the total luminosity and the number of ionising photons. More massive stars can clear out their direct surroundings more rapidly than less massive stars. Moreover, they provide stronger illumination, therefore, the neutral natal cloud material located far away will then become a reflection nebula. For less massive stars, hence less luminous, only their direct environment (e.g. circumstellar envelope) will be illuminated. In the final stages of the star's pre-main sequence evolution, the newly formed star will have removed most of its surrounding envelope, leaving only the remnant, dusty, accretion disk. These objects are known collectively as "isolated" Herbig Ae/Be stars (Waelkens \& Waters 1997).

The infrared spectra of Herbig Ae/Be stars reveal a wide variety of dust particles, including carbonaceous and (crystalline) silicate grains (van den Ancker et al. 2000; Bouwman et al. 2000; Waelkens et al. 1996). Specifically, many sources show IR emission around $3.3,6.2,7.6,7.8,11.2$, and $12.7 \mu \mathrm{m}$, attributed to polycyclic aromatic hydrocarbons (PAHs), i.e. large molecules of many fused aromatic rings (Leger \& Puget 1984; Cohen et al. 1985; Allamandola et al. 1989).

Isolated Herbig $\mathrm{Ae} / \mathrm{Be}$ stars often show evidence for the onset of planet formation (dust coagulation, settling) and even planet formation itself (in a few cases, Reipurth et al. 2007). 
Table 1. Stellar data.

\begin{tabular}{lllllllll}
\hline \hline Object & $\begin{array}{l}T_{\text {eff }} \\
{[\mathrm{K}]}\end{array}$ & $\log (g)$ & $\begin{array}{l}A_{\mathrm{v}} \\
{[\mathrm{mag}]}\end{array}$ & $\begin{array}{l}L_{\star} \\
{\left[L_{\odot}\right]}\end{array}$ & $\begin{array}{l}L_{\text {bol }} \\
{\left[L_{\odot}\right]}\end{array}$ & Spectral type & Group & $\begin{array}{l}\text { distance } \\
{[\mathrm{pc}]}\end{array}$ \\
\hline IRAS 06084 VLA1 & - & - & $\geq 40^{a}$ & - & $1 \times 10^{4 b}$ & ${\mathrm{~B} 0.5^{b}}^{b}$ & - & $1050^{c}$ \\
IRAS 06084 VLA4 & - & - & - & - & $1 \times 10^{3} b$ & $\mathrm{~B}^{b}$ & - & $1050^{c}$ \\
CD-42 11721 & 30000 & 3.88 & 5.08 & $8.71 \times 10^{3}$ & $1.05 \times 10^{3}$ & ${\mathrm{~B} 0 \mathrm{IVep}^{d}}^{c}$ & $\mathrm{I}$ & $400^{e}$ \\
TY CrA & 12000 & 4.07 & 2.02 & 30.9 & 27.5 & ${\mathrm{~B} 8 \mathrm{e}^{f}}^{g}$ & $?$ & $130^{g}$ \\
HD 176386 & 11000 & 3.94 & 0.62 & 49.0 & 21.4 & ${\mathrm{~B} 9 I V^{h}}^{f}$ & $?$ & $130^{f}$ \\
\hline
\end{tabular}

Note: $L_{\star}$ is the extinction corrected luminosity of the stellar photospheres, $L_{\mathrm{bol}}$ is the observed luminosity, including the infrared excess.

${ }^{a}$ Harvey et al. (1985); ${ }^{b}$ Gómez et al. (2000); ${ }^{c}$ Racine \& van den Bergh (1970); ${ }^{d}$ Shore et al. (1990); ${ }^{e}$ de Winter \& The (1990); ${ }^{f}$ Casey et al. (1998); ${ }^{g}$ Marraco \& Rydgren (1981); ${ }^{h}$ Houk \& Fuentes-Williams (1982).

Non referenced data have been obtained from Van den Ancker (private communication).

Based on the size of the infrared excess and where in the spectrum it starts to dominate, a sub-division is made in Group I and II sources (Meeus et al. 2001). In the latter case, the infrared emission is more modest. This difference has been attributed to differences in disk geometry (Meeus et al. 2001): Group I sources have flaring disks and Group II sources have more flattened disks with a shadowed region. PAHs are a good diagnostic for this geometry, as they emit through UVpumped fluorescence. Flaring disks subtend a larger solid angle viewed from the star and are thus able to reprocess more stellar light than flat disks.

The observed spectra reveal that PAHs undergo considerable processing during the early evolution of the YSO. Specifically, embedded object show a PAH spectrum resembling that of the ISM. More isolated Herbig Ae/Be stars show a more processed spectrum. These spectral variations imply considerable chemical changes, likely driven by the strong radiation field in the YSO's environment (Sloan et al. 2007; Boersma et al. 2008; Keller et al. 2008). Many aspects behind the processing, e.g. in what way the PAHs are modified, what process drives it, and when it commences, are not yet well understood. In this paper we compare spectra and images of four Herbig Ae/Be stars and speculate on the influence of stellar characteristics on the dust and morphology.

The paper has been organised as follows. In Sect. 2, the sources are introduced. In Sect. 3, the observations and data reduction are discussed. In Sect. 4 we describe our analysis and results. Subsequently, Sect. 5 presents a discussion and highlights the astronomical implications. Section 6 summarises our main conclusions.

\section{The sources}

$C D-4211721$ is a southern galactic star embedded in a small and extremely thick dark cloud. While it has been suggested that CD-42 11721 possibly is an evolved B[e] super-giant (e.g. Borges Fernandes et al. 2007, and references therein), its association with a star forming region as well as more general characteristics, e.g. its luminosity class (IV), establishes it in our view as a young stellar object.

IRAS 06084-0611 is a red nebulous object embedded in the Monoceros molecular cloud. Two bright mid-infrared sources are seen in the mid-IR images from Persi \& Tapia (2003) and are identified with the cometary HII region VLA1 (IRS 4) and the faint compact radio continuum source VLA4 (IRS 2). Persi \& Tapia (2003) suggest the latter is probably a Herbig Ae/Be star.

$T Y C r A$ is a multiple star-system consisting of at least four stars. Three stars are within $1.5 \mathrm{AU}$ of each other, of which one
Table 2. Collected data.

\begin{tabular}{lllll}
\hline \hline Source & TIMMI2 & VISIR & ISO & IRAC \\
\hline IRAS 06084 VLA1 & $\sqrt{ }$ & & $\sqrt{ }$ & $\sqrt{ }$ \\
IRAS 06084 VLA4 & $\sqrt{ }$ & & $\sqrt{ }$ & $\sqrt{ }$ \\
CD-42 11721 & $\sqrt{ }$ & & $\sqrt{ }$ & $\sqrt{ }$ \\
TY CrA & & $\sqrt{ }$ & $\sqrt{ }$ & $\sqrt{ }$ \\
HD 176386 & & $\sqrt{ }$ & & $\sqrt{ }$ \\
\hline
\end{tabular}

is a Herbig $\mathrm{Ae} / \mathrm{Be}$ star. The fourth star is located at a projected distance of $\sim 0.3^{\prime \prime}$ ( $40 \mathrm{AU}$ ) from the compact tertiary system. The three companions of the Herbig Ae/Be star are of later M-type (Chauvin et al. 2003). TY CrA is located near one of the densest parts of the R Corona Australis star-forming region, embedded in the bright reflection nebula NGC 6726/6727.

HD 176386 is an optically detected pre-main sequence star with a companion at $\sim 4$ " (i.e. CCDM J19017-3653B, Jeffers et al. 1963). The companion is likely a low-mass star (Turon et al. 1993). Also, signs of ongoing accretion have been reported (Grady et al. 2001). HD 176386 is located in the R Corona Australis star-forming region, $\sim 1^{\prime}$ from TY CrA.

The stellar characteristics of the four objects considered here are summarised in Table 1.

\section{Observations and reduction}

We have performed a $10 \mu \mathrm{m}$ spectral imaging study of the four Herbig Ae/Be stars introduced in Sect. 2. The Thermal Infrared MultiMode Instrument (TIMMI2) at the ESO $3.6 \mathrm{~m}$ telescope (Reimann et al. 2000) was used to obtain $N$-band imaging and spectroscopy of CD-42 11721 and the two bright mid-IR positions in IRAS 06084-0611 (VLA1 and VLA4). The Very large Imager and Spectrometer for the mid-IR (VISIR, Lagage et al. 2004) instrument at the Very Large Telescope (VLT) was used to obtain imaging and spectra of HD 176386 and TY CrA. The data are supplemented with data from the Short Wavelength Spectrometer (SWS, de Graauw et al. 1996) on board ESA's Infrared Space Observatory (ISO, Kessler et al. 1996) and from the InfraRed Array Camera (IRAC, Fazio et al. 2004) on board NASA's Spitzer space telescope. Table 2 summarises the data obtained for each target. The ground-based observation log is shown in Table 3.

\subsection{IRAC}

The InfraRed Array Camera (IRAC, Fazio et al. 2004) is a four channel camera providing simultaneous $5.2^{\prime} \times 5.2^{\prime}$ images at 3.6, $4.5,5.8$ and $8.0 \mu \mathrm{m}$. The detectors are $256 \times 256$ pixels in size, giving a pixel scale of $\sim 1.2^{\prime \prime}$. 
Table 3. Observation log of the TIMMI2 and VISIR data.

\begin{tabular}{lllllll}
\hline \hline Object & $\begin{array}{l}\text { RA }\left(^{\mathrm{h} \mathrm{m} \mathrm{s}}\right) \\
(\mathrm{J} 2000)\end{array}$ & $\begin{array}{l}\text { Dec }\left(^{\circ}{ }^{\prime \prime \prime}\right) \\
(\mathrm{J} 2000)\end{array}$ & Date & Calibrator star & Instrument & $\begin{array}{l}\text { Photometry } \\
{[\mathrm{Jy}]}\end{array}$ \\
\hline IRAS 06084 VLA1 & 061050.57 & -061149.7 & $2003-11-16$ & HD 39523 & TIMMI2 & 4.51 \\
IRAS 06084 VLA4 & 061050.32 & -061157.6 & $2003-11-17$ & HD 47205 & TIMMI2 & 1.72 \\
CD-42 11721 & 165906.78 & -424208.4 & $2005-02-25$ & HD 151249 & TIMMI2 & 6.63 \\
TY CrA & 190140.83 & -365233.9 & $2006-06-20$ & HD 178345 & VISIR & - \\
HD 176386 & 190138.93 & -365326.6 & $2007-08-29$ & HD 178345 & VISIR & - \\
\hline
\end{tabular}

(1) TIMMI2 $11.9 \mu \mathrm{m}$ (N11.9-OCLI filter) flux - determined as $2 \pi \sigma^{2} A$, where $\sigma$ and $A$ are the best fit values for the width and peak when fitting a 2D Gaussian to the source on the images.

A large portion of the $\mathrm{R}$ Corona Australis star-forming region and the Monoceros molecular cloud have been observed in the programme entitled "Structure and Incidence of Young Embedded Clusters" (PID 6). We retrieved the postBasic Calibrated Data (bcd; data that went through the extended pipeline) for these regions from the Spitzer Science Center (SSC) where it has been processed with pipeline version S14.0.0. From the High-Dynamical-Range (hdr; short exposure) mosaic, a $171 \times 171$ pixel sub-image was extracted around TY CrA and HD 176386. For IRAS 06084-0611, a $71 \times 71$ pixel sub-image was extracted around the positions of VLA1 and VLA4.

CD-42 11721 has been observed by the Spitzer Legacy programme GLIMPSE-Galactic Plane Survey (PID 192). Enhanced data products, made available by the GLIMPSE team, were retrieved and from the mosaics, a $171 \times 171$ pixel sub-image was extracted around CD-42 11721. Near the centre, the image appears saturated in two distinct peaks.

For each source, the 3.6, 5.8 and $8.0 \mu \mathrm{m}$ maps were combined into a single RGB-colour image, with the blue, green, and blue channel representing 3.6, 5.8, and $8.0 \mu \mathrm{m}$, respectively. The resulting images are presented in Fig. 1.

\section{2. $T I M M I 2$}

TIMMI2 has a camera that uses a $320 \times 240$ pixel Raytheon Si:As array. In imaging, the N11.9-OCLI filter was selected and a pixel scale of $0.2^{\prime \prime}$, providing a total field of view of $64^{\prime \prime} \times 48^{\prime \prime}$. For long-slit spectroscopy, the $10 \mu \mathrm{m}$ low-resolution grism was used. The grism covers wavelengths from 7.5 to $13.9 \mu \mathrm{m}$ and provides a spectral resolving power $\lambda / \Delta \lambda \sim 160$. The slit was $1.2^{\prime \prime} \times 70^{\prime \prime}$ in size and the pixel scale, $0.45^{\prime \prime}$. At the time of the observation, the slit could only be oriented North-South.

The slit was positioned across the two bright mid-IR components of IRAS 06084-0611, VLA1 and VLA4, and across the central star of CD-42 11721 (see Fig. 1). In order to correct for background emission from the sky, the observations were performed using a standard North-South chopping/nodding technique. The applied nod amplitude was the same as the chop amplitude, and the telescope nodded opposite to the chopping direction of the secondary mirror. The amplitude was $20^{\prime \prime}$ for IRAS 06084-0611 and 40" for CD-42 11721. These chop throws were large enough to avoid confusion.

The $N$-band imaging of the standard stars were performed in the same filter as the target and used for photometric flux conversion from photon count rates (ADU/s) into astronomical units (Jy), for atmospheric corrections and for establishing the point spread function (PSF). To minimise residuals of the telluric line cancellation, the standard star and the target were observed at similar air masses. A flux correction provided by the TIMMI2 instrument team takes care of the (small) differential air mass between the target and the standard star that remains. The synthetic calibrated spectra for the standard stars are taken from Cohen et al. (1999).

Data processing included the removal of bad frames and summing of all good chopping and nodding pairs. This procedure is applied to both target and calibrator star. For each pixel, the uncertainty, dominated by variations of the sky transparency, was propagated along each step of the processing. However, the chop amplitude of 40" used for CD-42 11721 turned out to be too large for the telescope mechanics. This resulted in a varying chop amplitude that introduced shifts of several pixels between the central position of the source on each frame, producing a chopping smearing along the North-South direction. This problem was solved by summing only the chopping and nodding pairs with a shift $\leq 1$ pixel. For imaging and spectroscopy this was 4 out of 48 and 196 out of 320 frames, respectively.

The spectra were extracted over apertures of 1.5 times the (average) full-width-at-half-maximum ( $F W H M)$ measured along the spatial direction, where the $F W H M$ for VLA1 and VLA4 are 4.7 and 3.6", respectively. The source profile of CD4211721 along the slit shows three emission peaks (see Figs. 1 and 2). Each of these peaks has been fitted with a Gaussian. These were then used to extract the spectrum of each component. In particular, the corresponding $F W H M$ of the central peak is $1.8^{\prime \prime}$ and $4.7^{\prime \prime}$ for both the bracketing peaks. The pixel-towavelength correspondence provided by the TIMMI2 support team is applied for wavelength calibration. The fully reduced spectra are presented in Figs. 3 and 4.

\subsection{VISIR}

VISIR has two DRS, former Boeing, $256 \times 256$ BIB detectors. In spectroscopy the pixel scale was set to $0.127^{\prime \prime}$. The low resolution $(\lambda / \Delta \lambda=250-390)$ configuration with a $1^{\prime \prime}$ slit was chosen to obtain spectra in the $8.8,9.8,11.4$ and $12.2 \mu$ m wavelength settings. A standard chop-nodding along the slit was applied to correct for background emission. The orientation of the slit was chosen such that, for TY CrA, both the "bar" and the central star are probed (see Fig. 1). For HD 17386 the orientation was chosen such that it encompasses the two emission sources visible in the $K$-band $(2.2 \mu \mathrm{m})$ acquisition image (see Fig. 1). Due to the extent of TY CrA and HD 176386 and their direct surroundings, chopping was done off the detector. Standard calibration has been done with calibrators with similar air masses at the time of observation. Detector Integration Times (DITs) were $0.0625 \mathrm{~s}$ for both the 8.8 and $9.8 \mu \mathrm{m}$ wavelength settings and 0.05 and $0.04 \mathrm{~s}$ for the 11.4 and $12.2 \mu \mathrm{m}$ wavelength settings, respectively. To improve the signal-to-noise, all four wavelength settings were observed two times $10 \mathrm{~min}$ for TY CrA, and four times 10 min for HD 176386.

The initial reduction steps are performed by the standard ESO reduction pipeline software, making use of the graphical 

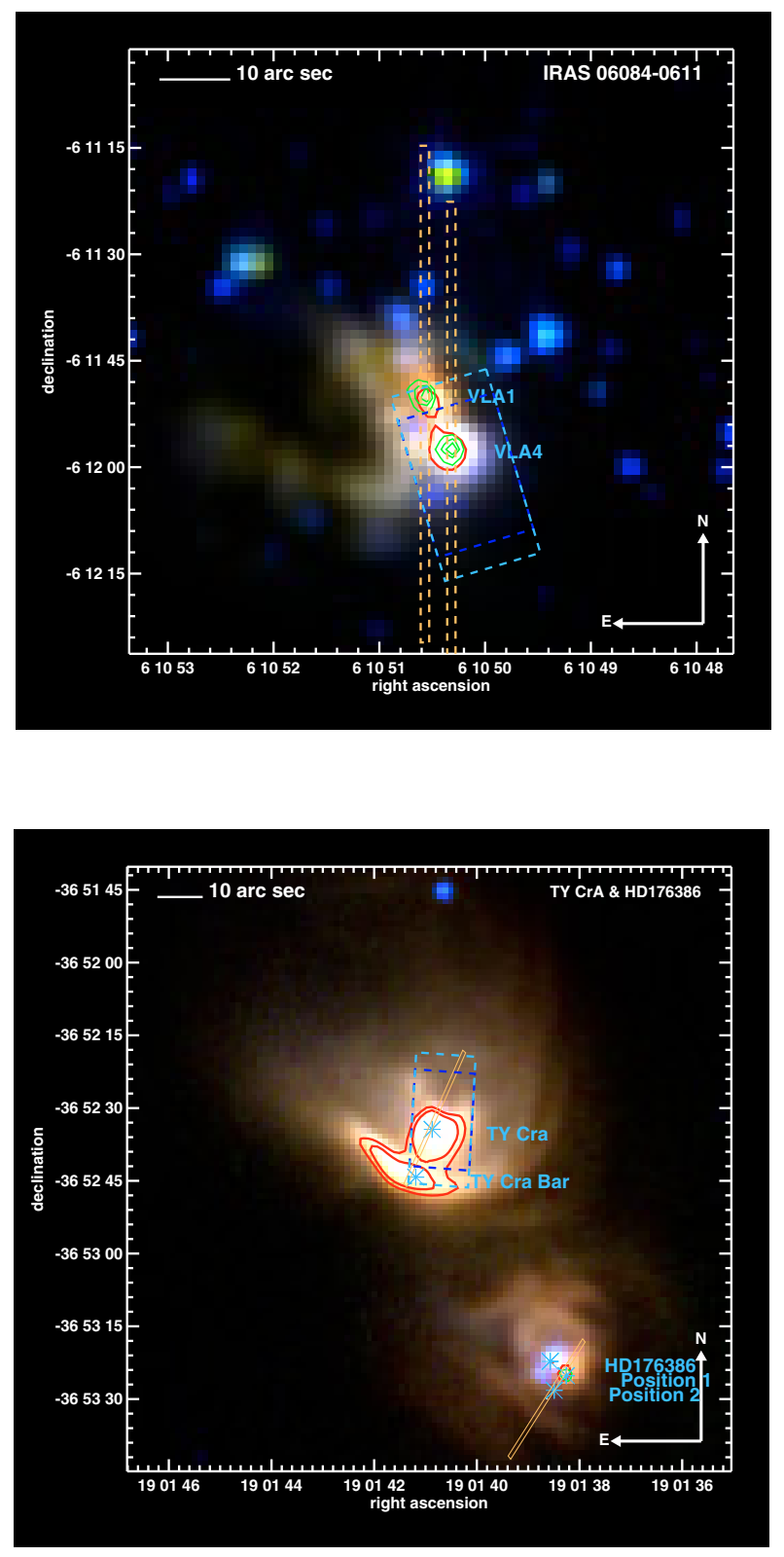

user interface to the pipeline, GASGANO. Pipeline products include the final, summed, distortion-corrected 2D spectrum for each wavelength setting, a pixel-to-wavelength map and a synthetic spectrum of the calibrator. The following reduction steps, are applied to the $2 \mathrm{D}$ spectra.

The residual background is corrected by fitting, ignoring the target's dispersion profile, each pixel row with a second order polynomial and subtracting it. Weight maps are constructed from the $2 \mathrm{D}$ spectra by collapsing them into the wavelength direction and forcing the area underneath the resulting spatial profile to one. The next step is multiplying the weight maps with the $2 \mathrm{D}$ spectra and collapsing the results in the spatial direction. The obtained 1D spectra are wavelength calibrated using the earlier obtained pixel-to-wavelength mapping. The target is flux calibrated by multiplying the target spectra by the synthetic spectra and dividing by the calibrator's spectra. Also, in view of the difference in chopping technique (off and on the slit), the number of beams on the detector is taken into account: two for the off-source chopped target and four for the calibrators. The four different wavelength settings are spliced to the mean of the overlapping area by multiplication by a factor, keeping the $9.8 \mu \mathrm{m}$

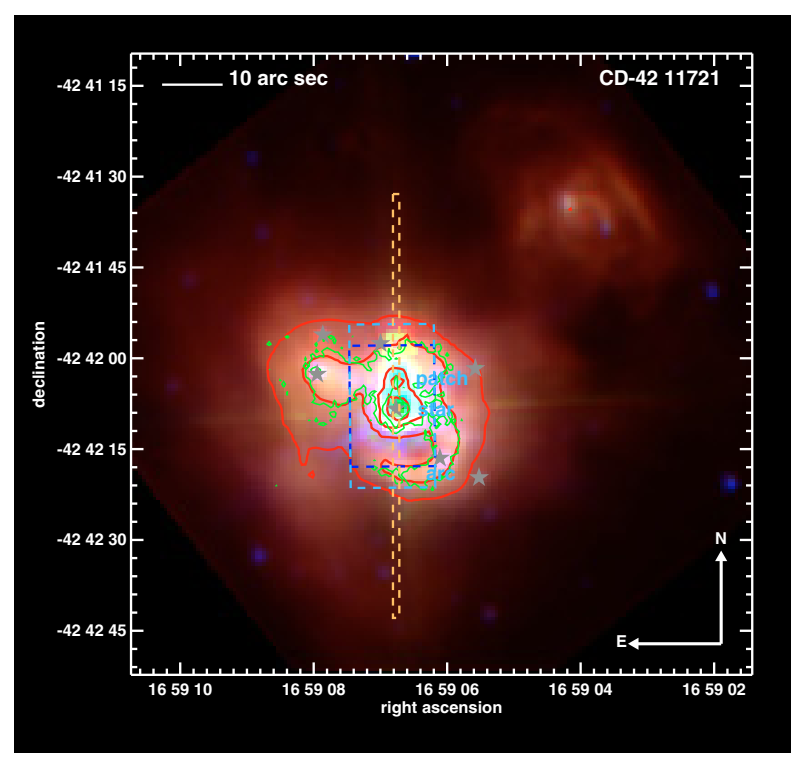

Fig. 1. Three colour composite IRAC images (red: 8.0, green: 5.8 and blue: $3.6 \mu \mathrm{m}$ ) of IRAS 06084-0611 positions VLA1 and VLA4, CD-42 11721 plus its surroundings and part of the R Corona Australis starforming region showing TY CrA, the TY CrA "Bar", and Position 1 and Position 2 of HD 176386, indicated by the blue asterisks. For IRAS 06084-0611 and CD-42 11721, the $8.0 \mu \mathrm{m}$ contours have been overlaid in red (@3250 MJy/sr and @ 1800 and $4200 \mathrm{MJy} / \mathrm{sr}$, respectively). For the same sources, the $11.9 \mu \mathrm{m}$ TIMMI2 data are overlain in green (@ 960, 1400 and $1800 \times 10^{4} \mathrm{MJy} / \mathrm{sr}$ and @ 130 and $350 \times 10^{4} \mathrm{MJy} / \mathrm{sr}$, respectively). For HD 176386, the VISIR $K$-band contours are shown in green (@ 35 ADU/s). In CD-42 11721, the grey stars represent 2MASS sources. The ISO SWS apertures are shown by dashed boxes, dark and light-blue for respectively wavelengths shortwards and longwards of $12 \mu \mathrm{m}$. The TIMMI 2 slits are indicated by the dashed orange lines, the VISIR slits by the solid orange lines.

wavelength setting constant. In the final reduction steps, all observations are combined. A correction for any uniform differences in flux is made by multiplying by a factor that brings the separate observations to the mean. The uncertainties are constructed by taking the statistical difference between all observations.

On top of the general reduction scheme described above, additional steps were required for TY CrA and HD 176386. Inspection of the (wavelength collapsed) spatial emission profile of TY CrA reveals two blended components in the source profile.

$$
I(x, \lambda)=A_{1}(\lambda) \mathrm{e}^{-\frac{\left(x-c_{1}\right)^{2}}{2\left(a_{1}+b_{1} \lambda\right)^{2}}}+A_{2}(\lambda) \mathrm{e}^{-\frac{\left(x-c_{2}\right)^{2}}{2\left(a_{2}+b_{2} \lambda\right)^{2}}}
$$

To disentangle both components we fit a function described by Eq. (1), where each diffraction pattern is assumed Gaussian and the wavelength dependent $F W H M$ of the pattern is taken linear $\left(a_{i}+b_{i}(\lambda)\right)$. At each wavelength bin $(\lambda)$, the diffraction pattern $(x)$ is fitted, determining the relative strength of the two components $\left(A_{1}(\lambda)\right.$ and $\left.A_{2}(\lambda)\right)$. Multidimensional minimisation is then performed on an entire wavelength setting to fix $a_{i}$ 


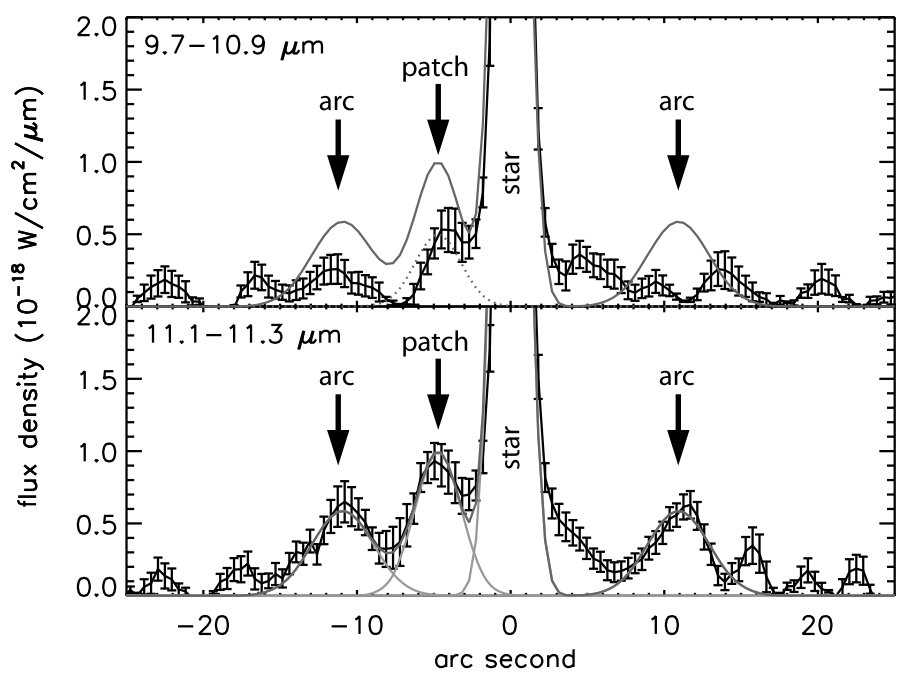

Fig. 2. Two spatial cross-cuts of the TIMMI2 spectra of CD-42 11721. The top panel plots the continuum between 9.7 and $10.9 \mu \mathrm{m}$, and the bottom panel plots the $11.2 \mu \mathrm{m}$ PAH band between 11.1 and $11.3 \mu \mathrm{m}$. Several wavelength rows have been collapsed to reduce the noise. The grey line (in both panels) represents a fit to the PAH cross-cut with 4 Gaussians. The dotted line in the top panel is a scaled version of the Gaussian peaking at $\sim-4.7^{\prime \prime}$. The PAH emission from the arc peaks around $\pm 11^{\prime \prime}$. Finally, the data reveals a separate, independent patch of emission which peaks around $\sim-5^{\prime \prime}$, near the central star.

and $b_{i}$. Figure 5 presents the extracted spectra for TY CrA. The established $F W H M$ as a function of wavelength for the PAH and amorphous silicate components is presented in Fig. 6.

The 2D spectral images of HD 176386 display two spatially separated sources. Hereafter these sources are referred to as Position 1 and Position 2. Position 1 corresponds to HD 176386 itself. For some observations, some wavelength settings show a strong varying background. Therefore, the Position 1 and 2 spectra are extracted by masking out the emission from the other position and following the reduction steps described above, but disregarding the wavelength settings where the background turns out to be too unstable for a proper extraction. Fortunately, for each wavelength setting there were at least two good observations, allowing for the construction of a complete spectrum. Figure 7 presents the spectrum of Position 1, median smoothed over 5 resolution elements.

\subsection{ISO-SWS}

ISO's SWS instrument provided medium and high spectral resolution in the wavelength region from 2.38 to $45.2 \mu \mathrm{m}$. For wavelengths smaller than $12 \mu \mathrm{m}$, the aperture on the sky was $14^{\prime \prime} \times 20^{\prime \prime}$ in size, for wavelengths between 12 and $27.5 \mu \mathrm{m} 14^{\prime \prime} \times 27^{\prime \prime}$. These apertures are indicated in Fig. 1.

All data were processed with $\mathrm{IA}^{3}$, the SWS Interactive Analysis package, using calibration files and procedures equivalent with pipeline version 10.1. A detailed account of the reduction can be found in Peeters et al. (2002b). Table 4 summarises the observations and the fully reduced spectra are presented in Fig. 8.

\section{Analysis and results}

For ISO-SWS data, PAH bands strengths are taken from Hony et al. (2001), van Diedenhoven et al. (2004) and Hony et al. (2001). When unavailable, these were calculated directly from

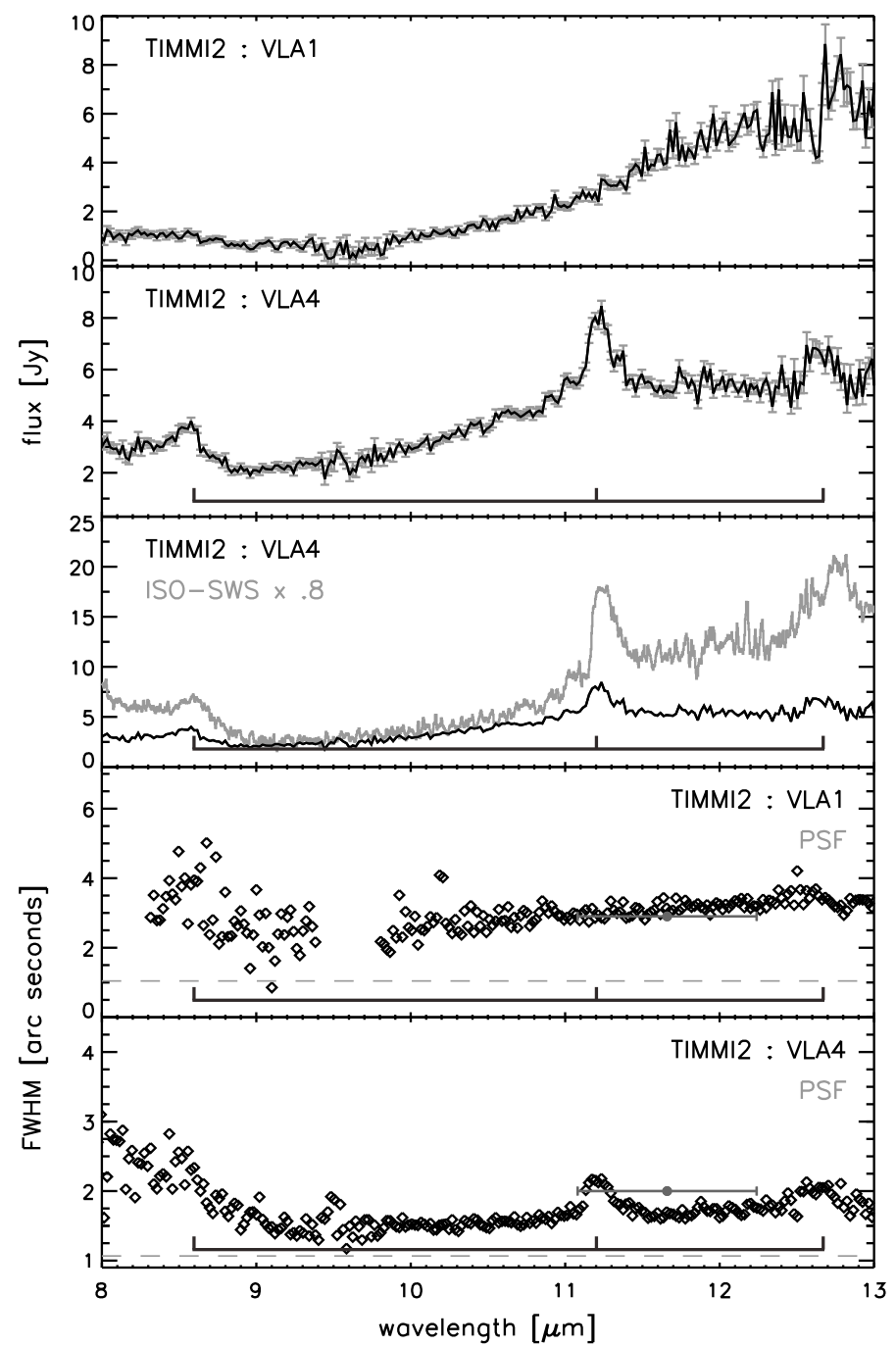

Fig. 3. The top two panels show the TIMMI2 spectra of IRAS 060840611 VLA1 and VLA4. A comparison between the ISO-SWS spectrum and the TIMMI2 spectra of IRAS 06084-0611 VLA4 (see Fig. 1 for the apertures) is plotted in the middle panel. The lower two panels shows the FWHM of both sources as a function of wavelength (black diamonds), the $F W H M$ of the sources in the $11.9 \mu \mathrm{m}$ image in the slit direction (grey dot) and the $F W H M$ of the calibration star (dashed grey line). Indicated are the 8.6, 11.2 and $12.7 \mu \mathrm{m}$ PAH bands.

the ISO-SWS spectrum following the procedures outlined by these authors. The PAH band strengths are presented in Table 5.

The IRAC images display a wealth of structure (Fig. 1). Therefore, some confusion is likely present in the spectra from these sources. In the following subsections the extent of the different sources and emission components are determined. The results have been summarised in Table 6 .

\subsection{IRAS 06084-0611}

The 3-colour IRAC image shows a nebulous region encompassing both VLA1 and VLA4, with an arm stretching towards the North-Eastern direction (Fig. 1).

The TIMMI2 spectra show for both sources quite different behaviour (top two panels of Fig. 3). VLA1's spectral shape resembles that of an embedded YSO, whereas VLA4 shows a far richer spectrum with strong PAH features at 8.6, 11.2 and 


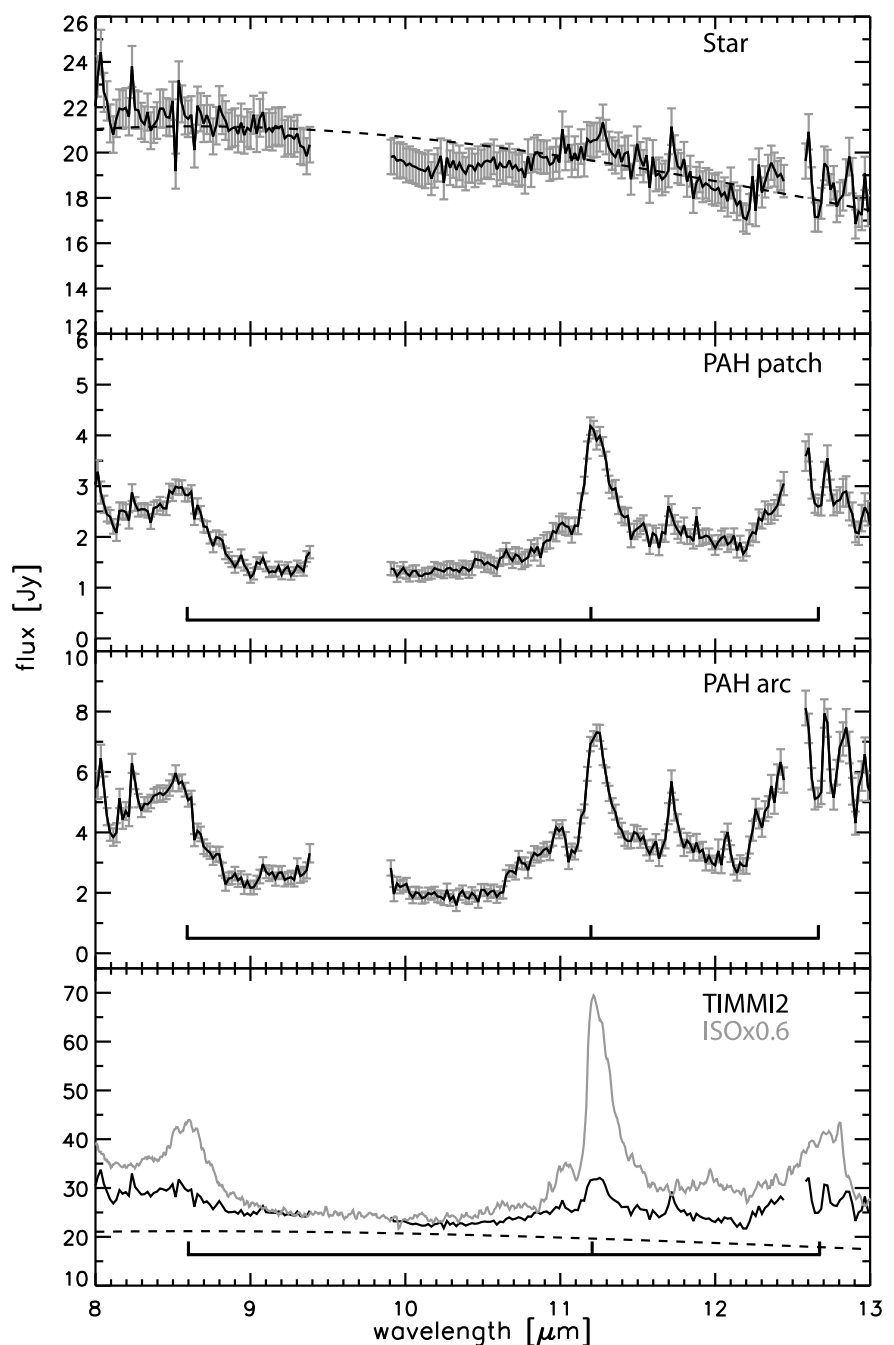

Fig. 4. The TIMMI2 spectra of the three components of CD-42 11721 (see Figs. 1 and 2, and Sect. 3.2) are shown in the top three panels. The fourth panel shows a comparison of the TIMMI2 spectrum of the four emission peaks combined with the ISO-SWS spectrum. The dashed lines show the fit made to the $\operatorname{star}(\log (F)=a+b \times \lambda$ with $a=-15.201 \pm$ $0.008, b=-0.1006 \pm 0.0008$ and $F$ in $\left.\mathrm{W} \mathrm{cm}^{-2}\right)$. Indicated are the 8.6, 11.2 and $12.7 \mu \mathrm{m}$ PAH bands.

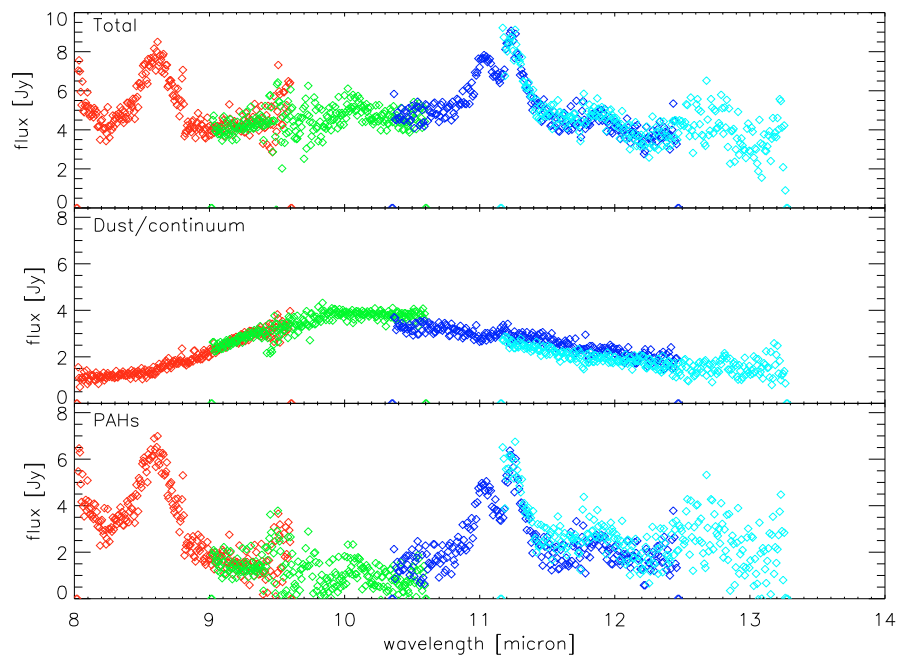

Fig. 5. Spectra for the total, amorphous silicate and PAH component of TY CrA when integrating the area underneath the Gaussians fitted to the spatial profile (see text for details).

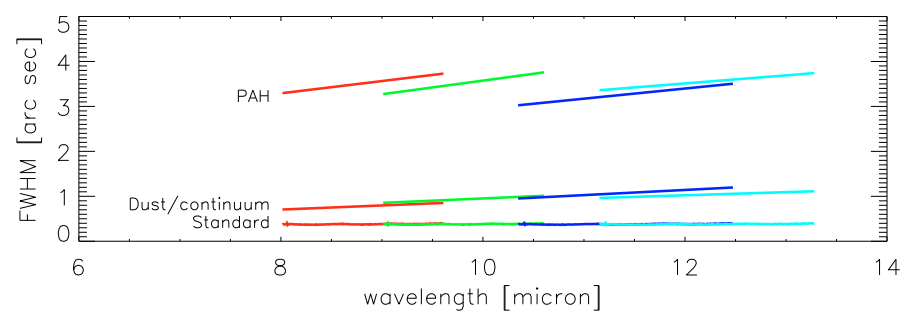

Fig. 6. $F W H M$ as a function of wavelength of the amorphous silicate and PAH components in TY CrA. Also shown is the FWHM of the calibrator as a function of wavelength.

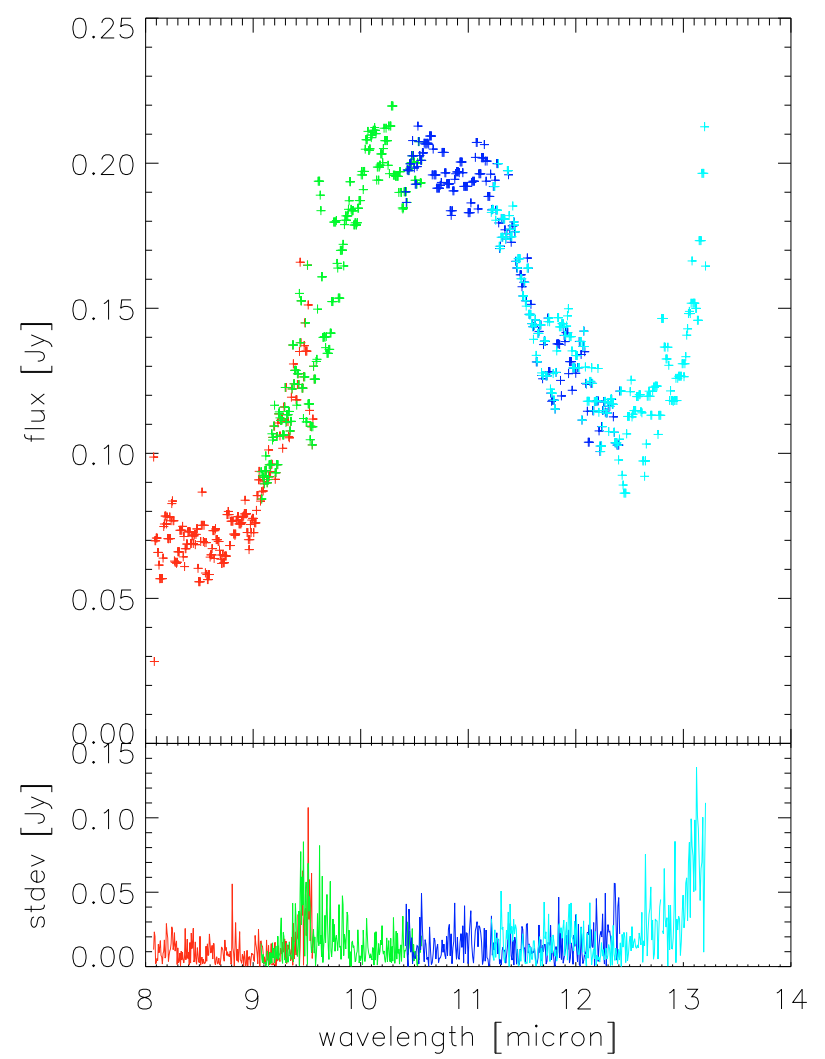

Fig. 7. VISIR spectrum of HD 176386 Position 1, median smoothed over 5 elements.

$12.7 \mu \mathrm{m}$. The ISO-SWS spectrum also shows these PAH features, in addition to those at 3.3, 6.2 and "7.7" $\mu \mathrm{m}$ (Fig. 8).

The short wavelength ISO-SWS aperture only covers VLA4, while the long wavelength ISO-SWS aperture encompasses both VLA1 and VLA4. Comparison of the ISO-SWS observations with the TIMMI2 observations centred on VLA4 is done in the third panel of Fig. 3. The apparent flux difference reflects the extent of the emission and the difference in aperture/slit size between TIMMI2 and ISO-SWS. The similarity between the continuum in the ISO-SWS spectrum and the TIMMI2 spectrum of VLA1 is striking and suggests a contribution of VLA1 to the ISO-SWS spectrum of VLA4 (Fig. 3).

The ISO-SWS spectrum also shows the $15.25 \mu \mathrm{m} \mathrm{CO}$-ice feature, which we attribute to absorption by ice grains along the line of sight towards the deeply embedded and very red object VLA1. Figure 9 presents the absorption profile in detail. Following the procedure outlined by Boogert (1999, Chapter 5 of his thesis), we obtain an ice temperature of 100-110 K, typical for an embedded YSO. 
Table 4. Summary of the ISO-SWS observations.

\begin{tabular}{cccc}
\hline \hline Source & $\begin{array}{c}\text { Pointing } \\
(\mathrm{J} 2000)\end{array}$ & $\begin{array}{c}\text { Template } \\
\text { (speed })\end{array}$ & $\begin{array}{c}R \\
(\lambda / \Delta \lambda)\end{array}$ \\
\hline IRAS 06084-0611 & $\alpha: 061050.17$ & AOT01 (3) & $\sim 1000$ \\
& $\delta:-061201.01$ & & \\
CD-42 11721 & $\alpha: 165906.79$ & AOT01 (2) & $\sim 450$ \\
& $\delta:-424207.99$ & & \\
TY CrA & $\alpha: 190140.70$ & AOT01 (3) & $\sim 1000$ \\
& $\delta:-365232.48$ & & \\
\hline
\end{tabular}

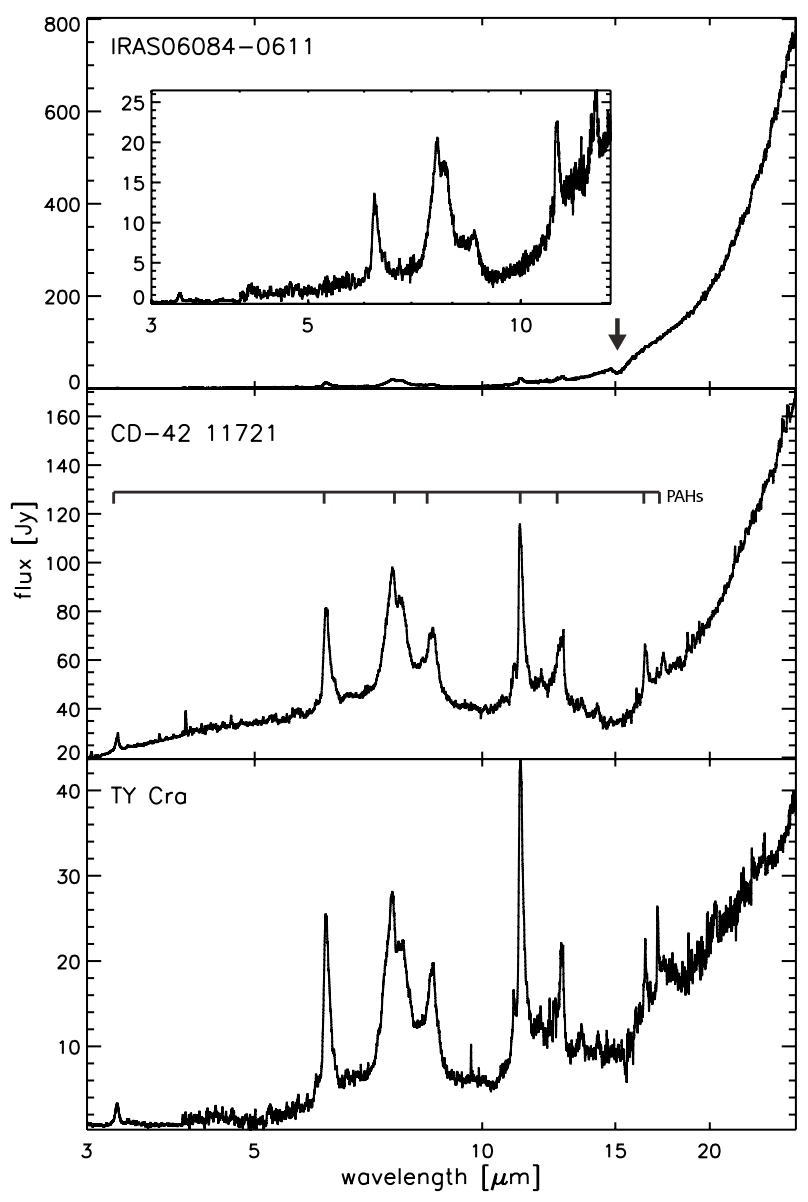

Fig. 8. ISO-SWS spectra of IRAS 06084-0611, CD-42 11721 and TY CrA. Indicated are the 3.3, 6.2, "7.7", 8.6, 11.2, 12.7, 16.4 and $17.4 \mu \mathrm{m}$ PAH bands. The arrow indicates the $15.25 \mu \mathrm{m} \mathrm{CO}_{2}$ bending mode, see Fig. 9 for a detailed profile.

To investigate the extent of the $10 \mu \mathrm{m}$ emission, the $F W H M$ as a function of wavelength is determined by fitting Gaussians to the spatial profiles on the calibrated 2D spectral images at every wavelength bin. The results are presented in the bottom two panels of Fig. 3 and are compared to the $F W H M$ of the calibrator. VLA1 is resolved and has a size of about $2.8^{\prime \prime}$ (2800 AU). VLA4 is also resolved and is more extended in the PAH bands $\left(\sim 2.2^{\prime \prime}\right.$; $2200 \mathrm{AU})$ than in the continuum $\left(\sim 1.2^{\prime \prime} ; 1300 \mathrm{AU}\right)$. From the $11.9 \mu \mathrm{m}$ TIMMI2 image, a FWHM of 2.9" (2900 AU) and 2.0" (2000 AU) is derived for VLA1 and VLA4, respectively. The sizes obtained from the images and spectra are in reasonable agreement.

In Fig. 10, the 11.2 and $10.5 \mu \mathrm{m}$ spatial profiles of VLA4 are compared to the $11.2 \mu \mathrm{m}$ spatial profile from the calibrator. Both the continuum $(9.7-10.9 \mu \mathrm{m})$ and the PAH emission $(11.1-11.31 \mu \mathrm{m})$ are resolved and show a skewed profile.
The continuum has an extent of 1700 AU (non-Gaussian $F W H M$ is $\left.1.6^{\prime \prime}\right)$ and the PAH emission extends over $2000 \mathrm{AU}$ (non-Gaussian $F W H M=1.9^{\prime \prime}$ ). The small difference in the extent between here and in Fig. 3 is likely due to the profile's deviation from a Gaussian.

\section{2. $C D-4211721$}

The 3-colour IRAC image shows a nebulous region (Fig. 1). Although the centre of the image is saturated, there appear two distinct emission peaks. One is the star, the other a close-by patch. Both peaks are separated by 4.3" (1700 AU). Additionally, an arc stretching towards the South-East is visible, which is also seen in the $11.9 \mu \mathrm{m}$ TIMMI 2 and the $8.0 \mu \mathrm{m}$ IRAC images. It is evident from the good agreement between the TIMMI2 and IRAC contours that the problems introduced by the varying chop throw (see Sect. 3) have been largely corrected for by our applied reduction steps (see Sect. 3.2). Note how the TIMMI2 slit cuts through both distinct emission peaks and the arc. These three components are also identifiable in the wavelength collapsed spatial cross-cuts of the TIMMI 2 spectra, Fig. 2. The separation between the central two emission peaks in the cross-cut $\left(\sim 4.0^{\prime \prime}\right)$ and the images are in reasonable agreement. Additionally, Fig. 2 possibly shows the arc extending onto larger scales, with its centre on the star and a projected radius of $\sim 11^{\prime \prime}$.

Summing all frames for the $11.9 \mu \mathrm{m}$ image obtained with TIMMI2, including those having shifts introduced by the chopping problem (see Sect. 3.2), the spatial $F W H M$ of the star is $\sim 4.7$ pixels $\left(0.94^{\prime \prime}\right)$. When only frames with a shift in pixels $\leq 1$ are considered, this $F W H M$ decreases to $\sim 3.9$ pixels $\left(0.78^{\prime \prime}\right)$, closer to the $F W H M$ of the standard stars (i.e. 2.9 pixels; $0.58^{\prime \prime}$ ) and therefore likely unresolved. The peaks of PAH emission are smeared out when all the frames are summed.

The ISO-SWS spectrum shows the prominent PAH features at 3.3, 6.2, "7.7", 11.2, 12.7 and $16.4 \mu \mathrm{m}$ (Fig. 8). The strengths of most of these bands are listed in Table 5. The TIMMI2 spectra for the four individual emission peaks are plotted in Fig. 4. The spectra of the PAH peaks are very similar, all showing the 8.6, 11.2 and $12.7 \mu \mathrm{m}$ PAH bands. In the fourth panel of Fig. 4, a scaled version of the ISO-SWS spectrum is compared to the total combined TIMMI2 spectrum (i.e. star + PAH components). The higher flux levels reflect the larger aperture of ISO-SWS and the extent of the PAH emission. The similar shaped spectrum of the arc and the nearby peak suggests that the same kind of material is radiating. As expected, the central star shows an almost featureless spectrum.

\subsection{TY CrA}

The 3-colour IRAC image shows a nebulous region surrounding TY CrA and a "bar" like structure to the South-East (Fig. 1).

From the VISIR 2D image we were able to decompose the central source profile into two components (see Sect. 3.3), separated by $\sim 2.5$ pixels $\left(0.32^{\prime \prime}\right)$. The two spectra extracted are shown in Fig. 5: a PAH spectrum and an amorphous silicate spectrum. The PAH spectrum, shown in the panel labelled PAHs of Fig. 5, clearly shows strong PAH bands at 8.6, 11.2 and $12.7 \mu \mathrm{m}$. Additionally, the shoulder of the "7.7" $\mu \mathrm{m}$ PAH band at the blue edge of the spectrum and the minor PAH features at 11.0 and $\sim 12 \mu \mathrm{m}$ are present. The ISO-SWS spectrum also shows these PAH bands, in addition to bands at 3.3, 6.2, "7.7", and $16.4 \mu \mathrm{m}$ (Fig. 8). The strengths of most of these bands are 
Table 5. PAH band strengths determined from the ISO-SWS spectra.

\begin{tabular}{llllllllllll}
\hline \hline Source & 3.3 & 6.0 & 6.2 & 7.6 & 7.7 & 7.8 & 8.6 & 11.0 & 11.2 & $F_{\text {¿PAH }}$ & Fir \\
\hline IRAS 06084-0611 & 1.2 & 0.61 & 15 & 24 & 33 & 7.7 & 1.7 & 0.44 & 6.6 & 0.046 & 31 \\
CD-42 11721 & 6.4 & 1.3 & 41 & 50 & 82 & 30 & 17 & 3.8 & 28 & 0.18 & 11 \\
TY CrA & 3.6 & 2.1 & 20 & 27 & 57 & 8.0 & 8.5 & 0.83 & 16 & 0.11 & 3.4 \\
\hline
\end{tabular}

Note: PAH band strengths are given in $10^{-14} \mathrm{~W} \mathrm{~m}^{-2} . F_{\text {IPAH }}$ and (Fir) are given in $10^{-11} \mathrm{~W} \mathrm{~m}^{-2}$.

Table 6. Gaussian $F W H M$ of the different objects and emission components studied here (still convolved).

\begin{tabular}{|c|c|c|c|}
\hline \multirow[t]{2}{*}{$\overline{\text { Band }}$} & \multicolumn{2}{|c|}{$\overline{F W H M[" / \mathrm{AU}]}$} & \multirow{2}{*}{$\begin{array}{l}\text { Spectroscopy/ } \\
\text { imaging }\end{array}$} \\
\hline & target & std. & \\
\hline \multicolumn{4}{|c|}{ IRAS 06084-0611 (VLA1), TIMMI2 } \\
\hline average & $4.7 / 4900$ & - & spectroscopy \\
\hline @ $10.0 \mu \mathrm{m}$ & $2.8 / 2900$ & $1.3 / 1400$ & spectroscopy \\
\hline @ $11.2 \mu \mathrm{m}$ & $2.8 / 2900$ & $1.1 / 1200$ & spectroscopy \\
\hline @ $11.9 \mu \mathrm{m}$ & $2.9 / 3000$ & $0.58 / 610$ & imaging \\
\hline \multicolumn{4}{|c|}{ IRAS 06084-0611 (VLA4), TIMMI2 } \\
\hline average & $3.6 / 3800$ & - & spectroscopy \\
\hline @ $10.0 \mu \mathrm{m}$ & $1.2 / 1300$ & $1.1 / 1200$ & spectroscopy \\
\hline @ $11.2 \mu \mathrm{m}$ & $2.2 / 2300$ & $1.3 / 1400$ & spectroscopy \\
\hline @ $11.9 \mu \mathrm{m}$ & $2.0 / 2100$ & $0.58 / 610$ & imaging \\
\hline \multicolumn{4}{|c|}{ CD-42 11721 (star), TIMMI2 } \\
\hline average & $1.8 / 720$ & - & spectroscopy \\
\hline @ $11.9 \mu \mathrm{m}$ & $0.94 / 380$ & $0.58 / 230$ & imaging (all frames) \\
\hline @ $11.9 \mu \mathrm{m}$ & $0.78 / 300$ & $0.58 / 230$ & imaging (shifted) \\
\hline \multicolumn{4}{|c|}{ CD-42 11721 (patch+arc), TIMMI2 } \\
\hline average & $4.7 / 1900$ & - & spectroscopy \\
\hline \multicolumn{4}{|c|}{ TY Cra (PAH) } \\
\hline @ $10.0 \mu \mathrm{m}$ & $3.0 / 390$ & $0.3 / 39$ & spectroscopy \\
\hline @ $11.2 \mu \mathrm{m}$ & $3.5 / 460$ & $0.3 / 39$ & spectroscopy \\
\hline \multicolumn{4}{|c|}{ TY Cra (Dust/continuum), VISIR } \\
\hline @ $10.0 \mu \mathrm{m}$ & $1.0 / 130$ & $0.3 / 39$ & spectroscopy \\
\hline @ $11.2 \mu \mathrm{m}$ & $1.4 / 180$ & $0.3 / 39$ & spectroscopy \\
\hline \multicolumn{4}{|c|}{ HD 176386, VISIR } \\
\hline $8-13 \mu \mathrm{m}$ & $\leq 0.3 / 39$ & $0.3 / 39$ & spectroscopy \\
\hline$K$-band & $0.52 / 68$ & - & imaging \\
\hline
\end{tabular}

listed in Table 5. The amorphous silicate spectrum peaks near $9.8 \mu \mathrm{m}$, indicative of the $\mathrm{Si}-\mathrm{O}$ stretch in amorphous silicate. Following van Boekel et al. (2005), the ratio of the silicate feature peak/continuum is $\sim 3.26$ and the $11.3 / 9.8 \mu \mathrm{m}$ ratio $\sim 0.74$.

The FWHM as a function of wavelength shows that both components are resolved and extended, Fig. 6. The PAH component extends over $\sim 3^{\prime \prime}(\sim 390 \mathrm{AU})$ and the amorphous silicate component over $\sim 1^{\prime \prime}(\sim 130 \mathrm{AU})$ in the spectra. On a larger scale, the spatial extent of the emission coming from TY CrA is studied by constructing a calibrated $2 \mathrm{D}$ spectrum. The different wavelength settings have been shifted, spliced and merged to form a continuous 2D spectrum. The results are shown in Fig. 11. Note that close to the blue and red cut-off wavelengths of the grating, the terrestrial background varies strongly with ambient conditions and atmospheric corrections are more difficult. The same holds for the wavelength regions of strong telluric lines near $9.58,11.73$ and $12.55 \mu \mathrm{m}$. Therefore, the quality of the $2 \mathrm{D}$ spectrum is lower in those regions. At $\sim 11^{\prime \prime}$ from TY CrA, the $11.2 \mu \mathrm{m}$ PAH emission from the "bar" to the South-East, is detected. The zoom-in (lower panel) reveals particularly well the extended nature of the 11.0 and $11.2 \mu \mathrm{m}$ PAH features.

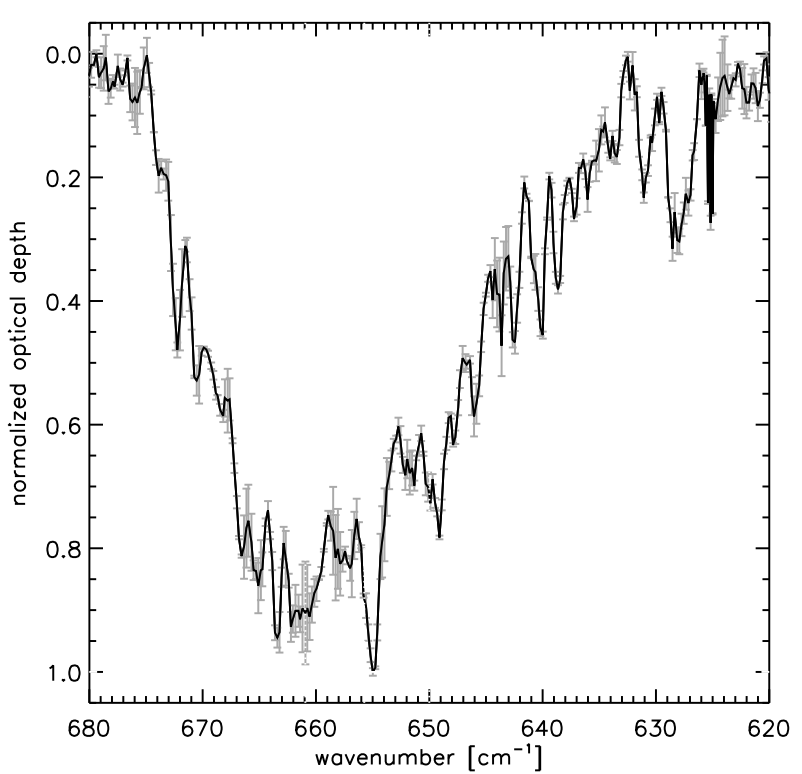

Fig. 9. $15.25 \mu \mathrm{m} \mathrm{CO}$ bending mode observed by ISO in IRAS 060840611. The uncertainties are indicated in grey.

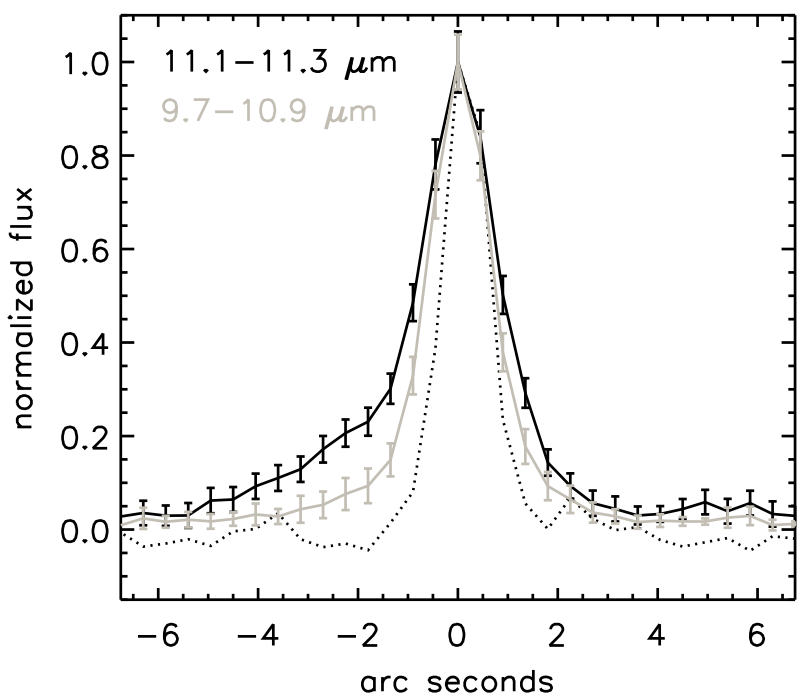

Fig. 10. Spatial distribution of IRAS 06084-0611 VLA4 (solid line) and the calibration star (dotted line) from TIMMI2 at $11.22 \mu \mathrm{m}$ (black). This is compared to the spatial distribution at $10.5 \mu \mathrm{m}$ (grey). For the continuum, the pixel rows from 9.7 to $10.9 \mu \mathrm{m}$ were summed; for the PAH band, pixel rows from 11.1 to $11.3 \mu \mathrm{m}$ were summed.

Given the extended nature of the mid-IR emission, spectra have been extracted using the PSF, determined from the $12.2 \mu \mathrm{m}$ wavelength setting, as extraction profile at positions along the slit, separated by half the PSF's FWHM. Zero is at the centre position of the slit, positions increase into the South-Eastern direction, see Fig. 1. At each position, the continuum is established by linear interpolation and subtracted from the PAH 


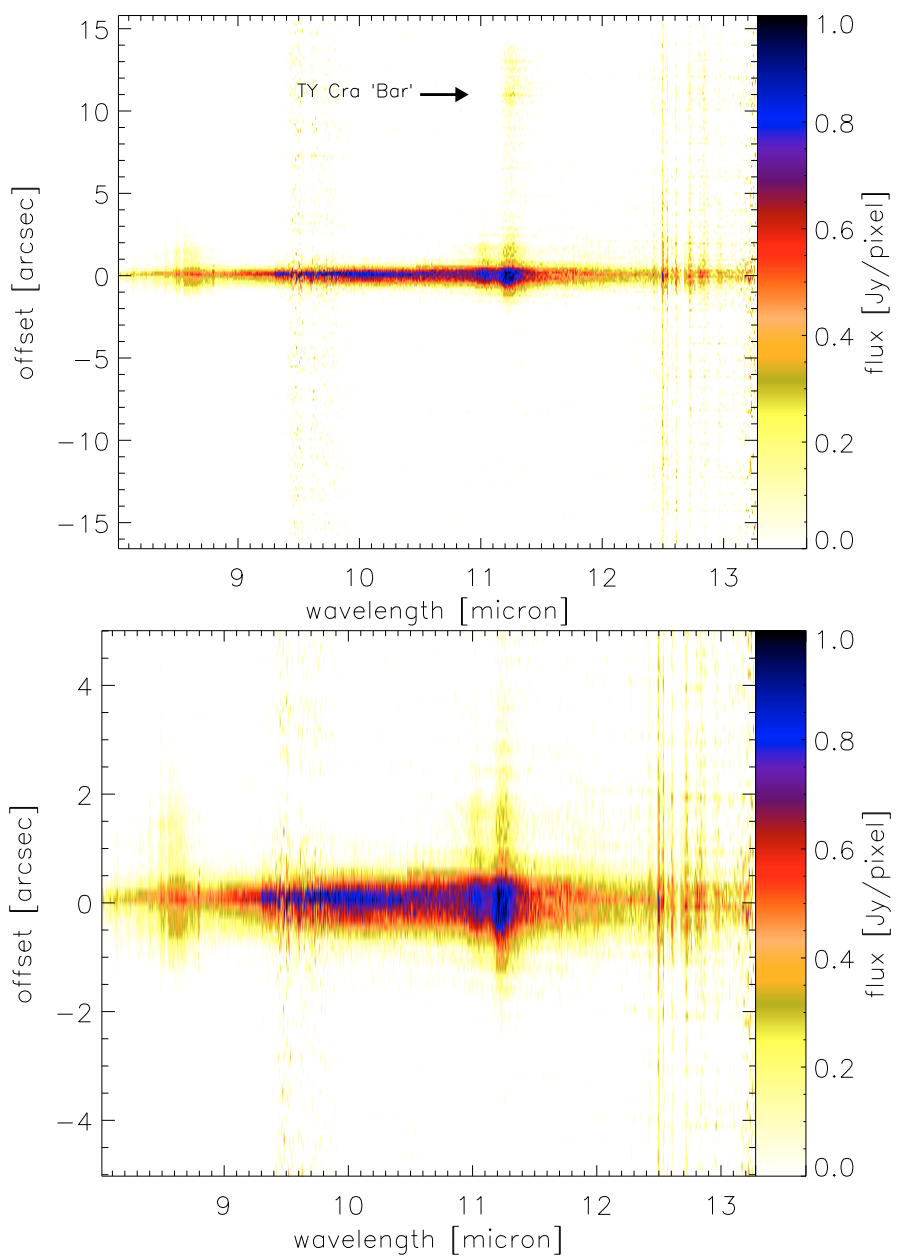

Fig. 11. 2D spectral image of TY CrA. Top: entire field of view (note the $11.2 \mu \mathrm{m}$ emission at $+11^{\prime \prime}$, this is the TY CrA "Bar"). Bottom: zoom-in on the dispersion profile, revealing the extent of the $11.0 \mu \mathrm{m}$ PAH band particularly well.

bands. For the $8.6 \mu \mathrm{m}$ band, the resulting profile is fitted with a single Lorentzian. The 11.0 and $11.2 \mu \mathrm{m}$ bands are decoupled by simultaneously fitting a Gaussian for the $11.0 \mu \mathrm{m}$ profile and a Lorentzian for the $11.2 \mu \mathrm{m}$ profile. This combination of Lorentzians and Gaussians was chosen because it provided the best fit with respect to the other possible combinations. The centres and widths of the profiles are allowed to vary marginally. The fit is assumed to be of intrinsically good quality. Therefore, uncertainties have been established by multiplying the diagonal co-variance matrix elements by the reduced $\chi^{2}$. We expect that relative variations along the slit are not much affected by systematic uncertainties introduced by the fitting procedure. The strength of the dust/continuum at each position is determined by integrating the emission between 9.7 and $10.6 \mu \mathrm{m}$. Here no error analysis has been performed. The results are presented in Fig. 12.

The spatial distribution of the individual components mostly differ from each other, but do also show some common characteristics. All spatial profiles show a higher flux level after the peak than before and an asymmetric flank to the right of it. The 8.6 and $11.0 \mu \mathrm{m}$ profiles are double peaked, whereas the $11.2 \mu \mathrm{m}$ and continuum profiles are single peaked. Individual peaks show a slight displacement from each other, traced out by the dashed lines in Fig. 12. The (non-Gaussian) FWHM varies as 4.8", $2.9^{\prime \prime}, 3.8^{\prime \prime}, 2.7^{\prime \prime}$ and $1.4^{\prime \prime}$ for the 8.6, 11.0, 11.2, 8.2 $\mu \mathrm{m}$ and
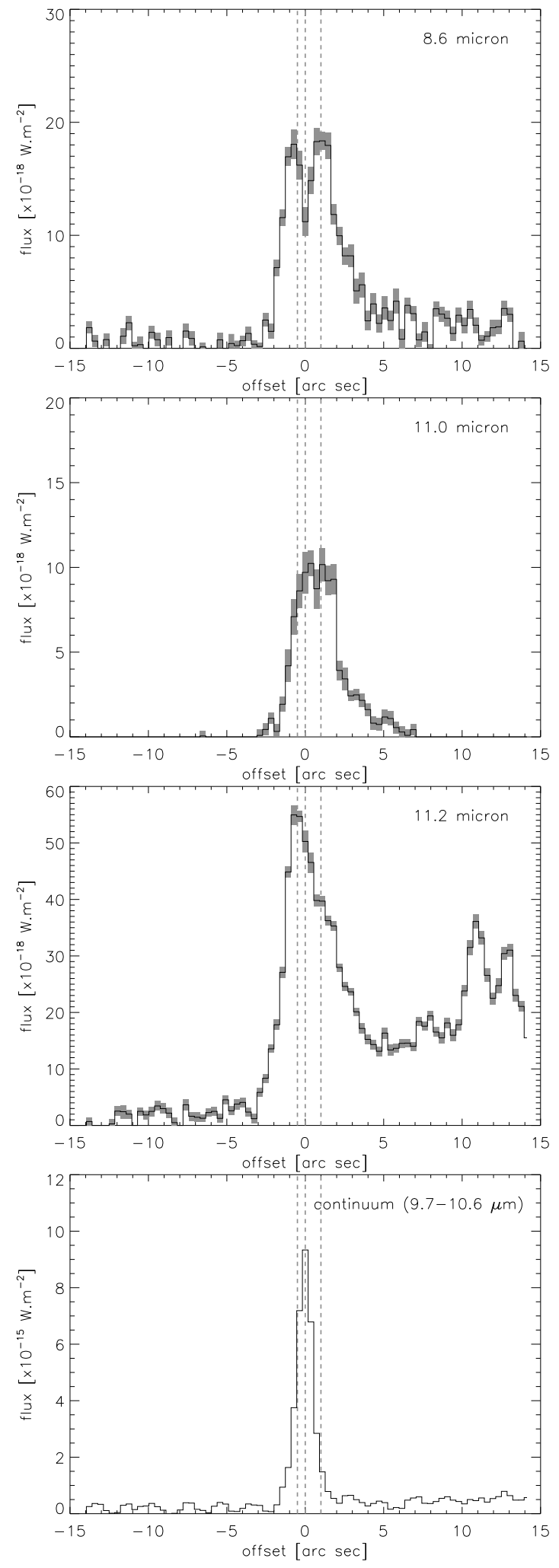

Fig. 12. Integrated band strength as a function of position for the 8.6, 11.0 and $11.2 \mu \mathrm{m}$ PAH features and the continuum in TY CrA. The dashed lines at $-0.5,0.0$ and $1.0^{\prime \prime}$ trace the $11.2 \mu \mathrm{m}$, continuum and second $8.6 \mu \mathrm{m}$ peaks, respectively, across all panels.

continuum profiles, respectively. These are in reasonable agreement with Fig. 6 (specially considering that the double peaked and asymmetric shape of the profiles are not well defined by a single Gaussian $F W H M$ ). The $11.2 \mu \mathrm{m}$ flux is particularly interesting in that it stays at a significant higher flux after peaking 


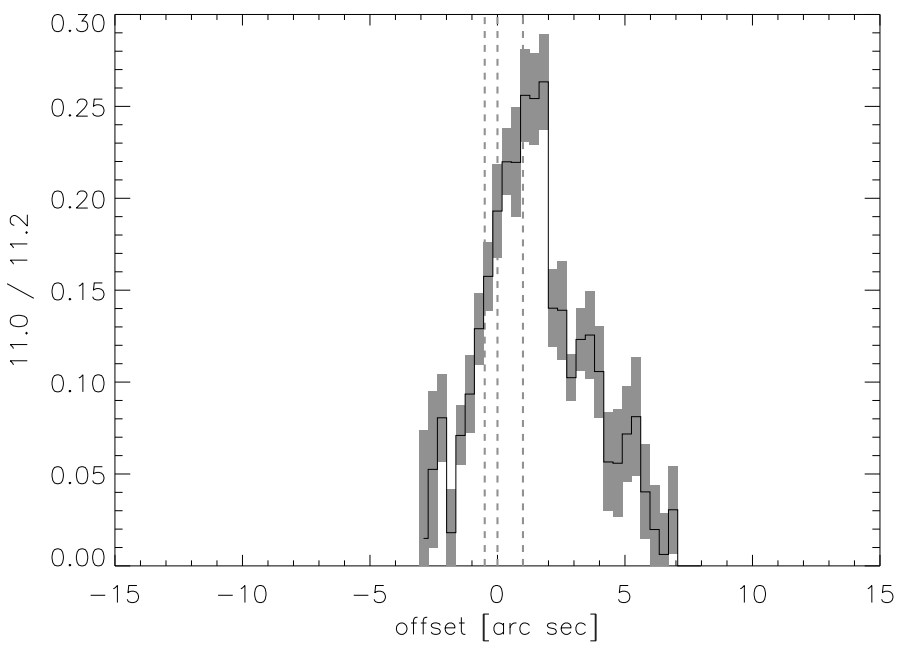

Fig. 13. 11.0/11.2 $\mu \mathrm{m}$ PAH band strength ratios of TY CrA as a function of position. The dashed lines at $-0.5,0.0$ and $1.0^{\prime \prime}$ trace the $11.2 \mu \mathrm{m}$, continuum and second $8.6 \mu \mathrm{m}$ peaks from Fig. 12 , respectively.

and coming down at $\sim 5^{\prime \prime}$, and rising again to form a double peak around $12.5^{\prime}$, the position of the TY CrA "bar".

The varying $11.0 / 11.2 \mu \mathrm{m}$ band strength ratio as a function of position is taken and presented in Fig. 13. The ratio rises steeply and peaks around 2 ", after which it drops sharply and continues dropping steadily until reaching zero around $7^{\prime \prime}$. The interpretation of these spatial distributions will be addressed in Sect. 5 .

\section{4. $H D 176386$}

The 3-colour IRAC image presented in Fig. 1 shows a nebulous region surrounding HD 176386. The $8.0 \mu \mathrm{m}$ IRAC contours are in good agreement with the VISIR $K$-band contours.

Position 2 is a star identified as CCDM J19017-3653B; its featureless spectrum is not shown and/or discussed here. The spectrum of Position 1, shown in Fig. 7, has some ozone residuals. However, an amorphous silicate feature can clearly be seen. Following van Boekel et al. (2005), we find a value of about 2.47 for the peak/continuum ratio, and about 1 for the $11.3 / 9.8 \mu \mathrm{m}$ ratio.

Concerning the extent of the emission, Position 1 and Position 2 are not resolved with VISIR in spectroscopy. On the $K$-band acquisition image, both sources have a $F W H M$ of about $0.52^{\prime \prime}$ ( $\left.68 \mathrm{AU}\right)$. Due to the unavailability of a $K$-band image of a point source calibrator we are unable to state whether the source is resolved. With spectroscopy, we derive an upper limit of $0.3^{\prime \prime}$ (40 AU), which is the $F W H M$ of the calibrator.

\section{Discussion and astronomical implications}

\subsection{Silicate dust}

The shape of the silicate feature is a measure of the amount of processing that the material has undergone (Bouwman et al. 2001). van Boekel et al. (2005) show that the amount of crystallisation can be obtained from the $11.3 / 9.8 \mu \mathrm{m}$ peak ratio. The peak/continuum ratio measures the feature's strength and correlates with the $11.3 / 9.8 \mu \mathrm{m}$ peak ratio. This correlation is interpreted as a relation between grain growth and crystallisation, which assesses the amount of processing the material has undergone. The values found for TY CrA and HD 176386 fall on the correlation these authors present. Both these sources show silicate emission originating from a disk; this is not the case for IRAS 06084-0611 VLA4 and CD-42 11721. The resulting 11.3/9.8 $\mu$ m peak ratios indicate that TY CrA and HD 176386 have slightly processed dust. The slightly higher value found for HD 176386 indicates more extensive processing. Assuming similar systems, this might suggest that HD 176386 is older. However, this is not really justified given that TY CrA is a complex multiple star system. Furthermore, the detailed evolutionary scenario involved in dust settling, grain growth and crystallisation is not yet fully understood. A mineralogical decomposition, which is beyond the scope of this paper, might provide further insight.

\subsection{PAHs}

Three out of the four Herbig Ae/Be stars studied here show PAH emission. The PAH profiles peak near 6.2, 7.6, and $11.2 \mu \mathrm{m}$, referred to by Peeters et al. (2002a, 2003) as class A, and are consistent with non-isolated Herbig Ae/Be stars (Peeters et al. 2002a). Class B profiles, with the PAH bands peaking closer to $6.3,7.8$ and $11.3 \mu \mathrm{m}$, are associated with isolated Herbig $\mathrm{Ae} / \mathrm{Be}$ stars and are thought to probe directly the circumstellar disk (Van Kerckhoven 2002; Boersma et al. 2008). Using the long-slit data, the measured extent $(F W H M)$ of the PAH emission is $\sim 2^{\prime \prime}(2100 \mathrm{AU})$ for VLA4 in IRAS06084-0611, $\sim 1^{\prime \prime}$ and 5" (400 AU and 2000 AU, respectively) for the patch near the star and arc components in CD-42 11721, and 3" (390 AU) for TY CrA. Given these sizes, it is clear that for VLA4 and CD-42 11721, the PAH emission from the surroundings is dominating the spectrum. For TY CrA, the emission associated with the central object likely originates from a large disk (scale size $\sim 170$ AU), while the more extended emission such, as the bar to the South-East, represents surrounding molecular cloud material set aglow by the radiation from the Herbig Ae/Be star.

\subsection{Morphology}

For HD 176386 (Position 1) we detect only silicate emission. This emission is not spatially resolved and therefore, given the scale, likely linked to a disk. The absence of PAH emission suggests a self-shadowed Group II disk. The fact that we do not detect any PAH emission, even at significant distances away from HD 176386, but do see it on the IRAC images is due to the limited sensitivity of VISIR compared to Spitzer. The estimated upper limit of the PAH emission from the IRAC $8.0 \mu \mathrm{m}$ image is $2 \times 10^{9} \times 0.3 \times 1.2 \times 2.35 \times 10^{-11} \simeq 0.017 \mathrm{Jy}\left(\mathrm{IRAC}_{\text {fluxin1pixel }}\right.$ $\left.[\mathrm{MJy} / \mathrm{sr}] \times \mathrm{PSF}_{\text {VISIR }}\left[{ }^{\prime \prime}\right] \times \operatorname{pixel}_{\text {IRAC }}\left[{ }^{\prime \prime}\right] \times \square / \mathrm{sr}\right)$, which is similar to the noise (see Fig. 7). Siebenmorgen et al. (2000), using ISOCAM, do detect PAH emission from HD 176386 and also from a nearby "bar"-like component. Due to the orientation of the VISIR slit, the "bar"-like component is not present in our data. Given the large aperture of ISOCAM, the PAH emission Siebenmorgen et al. (2000) see must be originating from the surrounding area.

The difference in spatial distribution of the 8.6, 11.0 and $11.2 \mu \mathrm{m}$ features in the spectra of TY CrA (Fig. 12) point towards three distinct carriers for these bands. The $8.6 \mu \mathrm{m} \mathrm{PAH}$ band has recently been attributed to very large, compact and highly symmetric PAH cations (and anions, Bauschlicher et al. 2008). The $11.0 \mu \mathrm{m}$ band has been assigned to the out-of-plane bending mode in solo $\mathrm{C}-\mathrm{H}$ cationic PAHs, while the $11.2 \mu \mathrm{m}$ band has been assigned to the out-of-plane bending mode in solo C-H of neutral PAHs (Hony et al. 2001; Hudgins \& Allamandola 

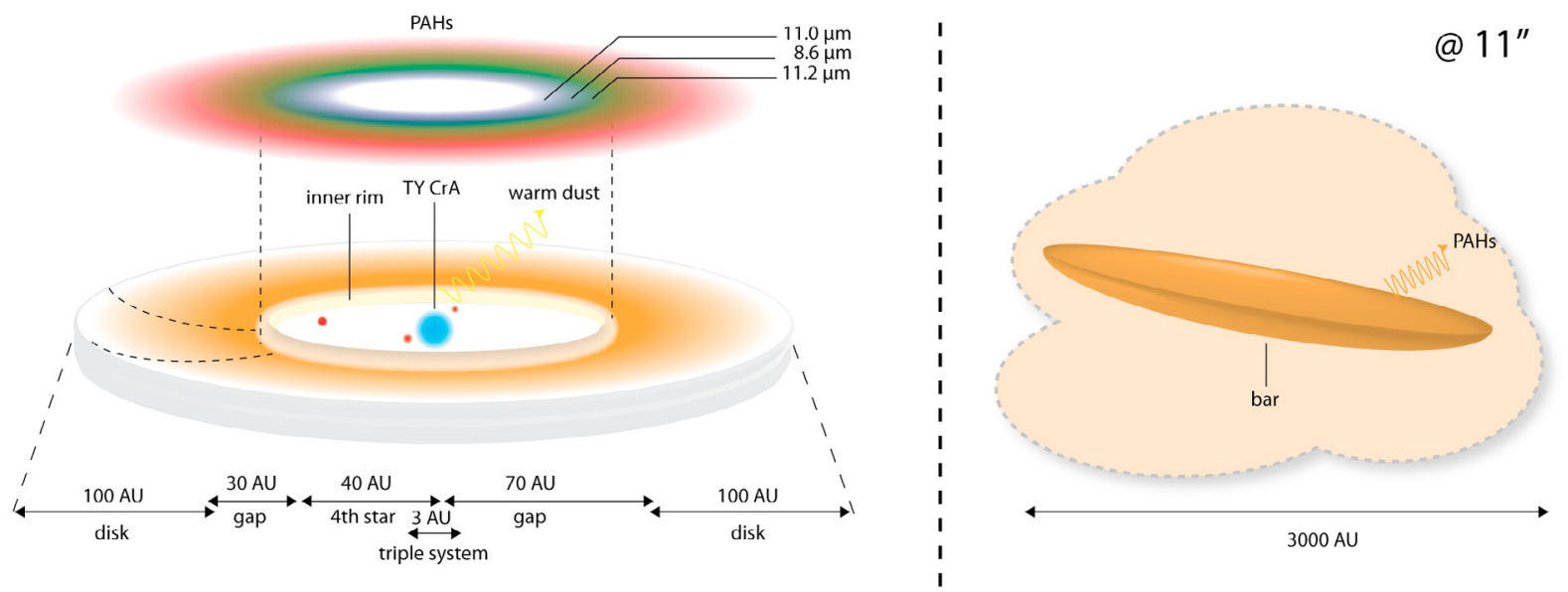

Fig. 14. Cartoon of the proposed morphology for TY CrA depicting the different emission zones.

1999). Although, recently this assignments has met some opposition (Povich et al. 2007).

Attributing the PAH emission originating from a flaring disk, the spatial distribution can be explained within the context of these assignments. The proposed morphology of TY CrA is presented in Fig. 14, where the quaternary star system is surrounded by a single flaring disk. An inner gap beyond the orbit of the fourth stellar component is inferred from the double peaked spatial profiles of the 8.6 and $11.0 \mu \mathrm{m}$ PAH bands. The 11.0/11.2 ratio - and thus the ions to neutrals - peaks to the right, away from the star and only to one side. This can be understood in the context of a flaring disk seen under an angle, where the near side is foreshortened and therefore unobserved. The $11.2 \mu \mathrm{m}$ emission originates from the bulk of the disk. Both the neutral and intrinsically stronger nature of the $11.2 \mu \mathrm{m}$ PAH band allows for this. The emission can even be traced all the way down to the TY CrA "bar", which we suspect to be the limb-brightened edge of a bowl where the photo dissociation front is eating its way into the molecular cloud. This in-between material is the non-limb brightened inside of the bowl. The PAHs will be more easily ionised close to the star. In agreement, the 8.6 and $11.0 \mu \mathrm{m} \mathrm{PAH}$ band emission is originating from a narrow region at the inner disk. Furthermore, recent analysis by Bauschlicher et al. (2008) suggest larger PAHs dominating the $11.0 \mu \mathrm{m}$ emission than those dominating that at $8.6 \mu \mathrm{m}$. Hence, the $11.0 \mu \mathrm{m}$ emission should be more confined since only large PAHs are able to survive close to the star, and this is what is seen. Geers et al. (2007) measured the radial extent of the $3.3 \mu \mathrm{m}$ PAH band emission to be $54 \mathrm{AU}$, which fits well within the proposed morphology.

Interestingly, the fourth stellar component is separated $\sim 0.3^{\prime \prime}$ (40 AU) from the central tertiary system, which is similar to the separation of the dust and PAH component in the VISIR spectrum. It is tempting to speculate that the fourth companion, at a projected distance of $40 \mathrm{AU}$, is dynamically related to the inferred large inner radius of the circumquartery disk. That would imply that the actual distance of this star is some $70 \mathrm{AU}$ from TY CrA itself. It is tempting to conclude that the fourth companion is inducing the dust emission. However, at this distance, the central Herbig Ae/Be star still dominates the dust temperature ( 300 Kelvin, assuming LTE and an emissivity of 0.05$)$. Furthermore, the M4 star is unable to heat the dust up to the scale of the observed distance ( $\sim 65 \mathrm{AU})$. Therefore, the central Herbig Ae/Be star must be responsible for heating the dust.
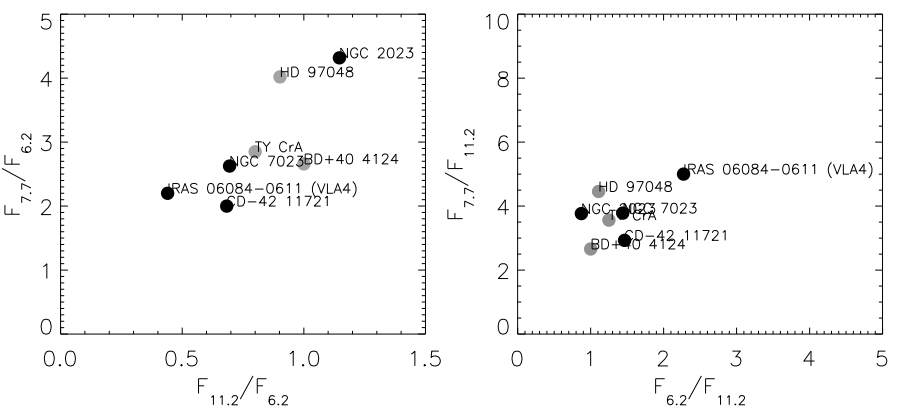

Fig. 15. Left: $F_{11.2 / 6.2}$ versus $F_{7.7 / 6.2} \mathrm{PAH}$ band strength ratio. Right: $F_{6.2 / 11.2}$ versus $F_{7.7 / 11.2} \mathrm{PAH}$ band strength ratio. The reflection nebula are shown in black and the Herbig Ae/Be stars in grey.

\subsection{Band strengths}

Following Hony et al. (2001) and Galliano et al. (2008), we have investigated the correlations between the strength of the PAH bands presented in Table 5. The 6.2/11.2 $\mu \mathrm{m}$ band shows little variations, implying very similar physical conditions. In contrast, other studies (Galliano et al. 2008; Lebouteiller et al. 2008) report little variation in the 7.7/6.2 ratios with a typical value of 2.5, where most of the Herbig stars fall (Fig. 15). There is no indication that this ratio correlates with object type (e.g. isolated Herbig stars versus reflection nebula). The origin of this variation may well provide interesting insight in the chemical makeup of the emitting PAHs, for example the difference in photochemical activity.

The 11.2/6.2 versus 7.7/6.2 $\mu \mathrm{m}$ band strength ratios have been plotted in Fig. 15. Along with the ratios for two reflection nebulae, NGC 7023 and NGC 2023, and two non-isolated Herbig Ae/Be stars, HD 97048 and BD+40 4124 (Hony et al. 2001; van Diedenhoven et al. 2004; Hony et al. 2001). These band ratios may be influenced by the physical conditions (e.g. charge) or by the chemical history (e.g. difference in illuminating spectrum may control photochemistry).

The ratio of the $\mathrm{C}-\mathrm{C}$ to $\mathrm{C}-\mathrm{H}$ modes $(6.2 / 11.2 \mu \mathrm{m}$ band strength ratio) is generally thought to reflect the degree of ionisation of the emitting PAHs (Tielens 2008). Typically, these ratios vary with a factor of $\sim 5$ between sources and within sources (Galliano et al. 2008). However as a class, these Herbig Ae/Be stars reveal very similar values (Fig. 15), indicating that the physical conditions (e.g. $G_{0} / n_{\mathrm{e}}$, where $G_{0}$ is the UV intensity 
and $n_{\mathrm{e}}$ the electron density) in the emission zone are much the same. Curiously in the same data, in terms of the 7.7/6.2 versus 11.2/6.2 band ratios, a larger variation is apparent (Fig. 15). In particular, the sources HD 97048 and NGC 2023 have a 7.7/6.2 ratio which is almost a factor 2 higher than in the other sources.

\subsection{PAH emission from Herbig $A$ vs Herbig $B$ stars}

The (proto-) stellar mass determines the total luminosity and the number of ionising photons. Heavier stars, and therefor more luminous, should be able to light up more of their surroundings than there less massive counterparts. At the same time the number of available ionising photons is larger for the heavier stars. Alterations to the silicates and PAH family, driven by photochemistry, should be sensitive to the spectral shape of the irradiating source and show up as variations in the band shapes.

Herbig B stars are more luminous analogs of the Herbig A stars. The earliest B star, which are powering prominent reflection nebulae (e.g. NGC 2023 and NGC 7023), have about a hundred times higher luminosity at $6 \mathrm{eV}$ than the later type Herbig B stars (e.g. TY CrA), while mid A stars are another 100 times weaker yet. Hence, typical spatial scales for the PAH emission will be ten times larger for early B stars than later Herbig B stars and a hundred times larger than mid Herbig A stars. Indeed, for reflection nebula spatial scales for PAH emission are $\sim 0.1 \mathrm{pc}$, whereas for Herbig A stars only the circumstellar disk re-radiates PAH emission ( $200 \mathrm{AU})$. In this context, IRAS 06084-0611 VLA4 and CD-42 11721 are placed in the RNe class and indeed their emission scale is large and consistent with surrounding molecular cloud material illuminated by the star, but not associated with it. TY CrA is possibly a transition object between the Herbig A and genuine reflection nebulae. The reflection nebula characteristics dominate on larger scales, while the disk and envelope of TY CrA dominate on smaller scales.

\section{Summary and conclusions}

The mid-IR images and spectra of four Herbig Ae/Be stars have been investigated in order to study the variation in the dust and PAH characteristics and hence, the processing of material in star and planet forming environments. We find evidence for large scale structure and extended emission in all four sources. Except for HD 176386, all sources show PAH emission in their TIMMI2/VISIR spectrum. Both HD 176386 and TY CrA show silicate emission. The scales on which these dust components emit varies significantly. The PAHs are detected from $390 \mathrm{AU}$ up to $5700 \mathrm{AU}$. The silicate emission observed in HD 176386 is spatially unresolved, providing an upper limit of 40 AU. In TY CrA, the silicates are seen up to $130 \mathrm{AU}$. The extent of the continuum emission is similar to that of the silicates.

Following Hony et al. (2001) and Galliano et al. (2008), we have investigated the correlation of the strength of the PAH bands. The $6.2 / 11.2 \mu \mathrm{m}$ PAH band ratio shows little variation, implying very similar physical conditions. In contrast to previous studies, we find more variation in the 7.7/6.2 $\mu \mathrm{m}$ band strength ratio within our sample. However there does not seem to be a relationship with object type.

For the individual objects we infer the following:

IRAS 06084-0611: The two bright IR sources VLA1 and VLA4 have been studied, where the spectrum of VLA1 resembles that of a YSO. For this source, we were able to deduce a $\mathrm{CO}_{2}$ temperature of $100-110 \mathrm{~K}$, consistent with an embedded YSO. VLA4 shows a far richer spectrum. The $\mathrm{PAH}$ and continuum emission is extended on scales of 2800 and $2200 \mathrm{AU}$, respectively.

CD-42 11721: We find evidence for several distinct emission components. First, there is evidence for a PAH peak close to the star. Second, an arc of PAH emission partially encompasses the star and the nearby emission peak ("patch"). The nearby PAH peak and PAH arc have both an emission scale of 1900 AU.

TY CrA: Our analysis was particularly successful given our ability to separate an amorphous silicate and PAH spectrum. The silicates and PAHs are seen up to scales of 130 and 390 AU, respectively. Extracting spectra separated 1 PSF over the source profile allowed us to infer the morphology of TY CrA. The TY CrA system is seen under an angle and includes a large circum-quaternary disk with a inner gap of $\sim 70$ AU. Analyses of the $10 \mu \mathrm{m}$ feature given the paradigm of van Boekel et al. (2005) suggests the presence of rather pristine silicates.

HD 176386: Spectra of two positions were acquired. One of them was identified as CCDM J19017-3653B and has a stellar spectrum. At the other position, a $10 \mu \mathrm{m}$ feature was detected. Following the analysis of van Boekel et al. (2005), pristine silicate were identified, but more processed than those of TY CrA. The absence of PAH emission is consistent with a self-shadowed Group II disk.

The variety in emission scales distinguishes two classes of objects. In the first, the morphology and spectral characteristics are close to those of reflection nebulae. In the second, the characteristics conform with those of Herbig A stars. This distinction reflects a luminosity difference. In Herbig B stars, the dust emission from the surroundings dominates, where for Herbig A stars, the disk dominates the emission. In this scheme, IRAS 06084-0611 and CD-42 11721 resemble reflection nebulae and HD 176386 a more typical Herbig Ae/Be star. TY CrA shows characteristics both common to genuine reflection nebulae and Herbig B stars.

A consistent large sample of high-spatial observations of reflection nebula and Herbig Ae/Be stars will help pin down the validity of our proposed connection between Herbig A and B stars and reflection nebulae and determine how smooth the transitions actually are. Such a study could form the basis for our understanding of the evolution of PAHs in regions of star and planet formation.

Acknowledgements. We are grateful to the staff at the European Southern Observatory in La Silla and Paranal for assisting with the observations. We would also like to extend our gratitude to Mario van den Ancker for providing an overview of the stellar data. We also thank Carsten Dominik for insights concerning disk geometry.

\section{References}

Allamandola, L. J., Tielens, G. G. M., \& Barker, J. R. 1989, ApJS, 71, 733 Bauschlicher, Jr., C. W., Peeters, E., \& Allamandola, L. J. 2008, ApJ, 678, 316 Boersma, C., Bouwman, J., Lahuis, F., et al. 2008, A\&A, 484, 241 Boogert, A. C. A. 1999, Ph.D. Thesis, AA

Borges Fernandes, M., Kraus, M., Lorenz Martins, S., \& de Araújo, F. X. 2007, MNRAS, 377, 1343

Bouwman, J., de Koter, A., van den Ancker, M. E., \& Waters, L. B. F. M. 2000, A\&A, 360, 213

Bouwman, J., Meeus, G., de Koter, A., et al. 2001, A\&A, 375, 950

Casey, B. W., Mathieu, R. D., Vaz, L. P. R., Andersen, J., \& Suntzeff, N. B. 1998, AJ, 115, 1617 
Chauvin, G., Lagrange, A.-M., Beust, H., et al. 2003, A\&A, 406, L51

Cohen, M., Tielens, A. G. G. M., \& Allamandola, L. J. 1985, ApJ, 299, L93 Cohen, M., Walker, R. G., Carter, B., et al. 1999, ApJ, 117, 1864

de Graauw, T., Haser, L. N., Beintema, D. A., et al. 1996, A\&A, 315, L49 de Winter, D., \& The, P. S. 1990, Ap\&SS, 166, 99

Fazio, G. G., Hora, J. L., Allen, L. E., et al. 2004, ApJS, 154, 10

Galliano, F., Madden, S. C., Tielens, A. G. G. M., Peeters, E., \& Jones, A. P. 2008, ApJ, 679, 310

Geers, V. C., van Dishoeck, E. F., Visser, R., et al. 2007, A\&A, 476, 279

Gómez, Y., Rodríguez, L. F., \& Garay, G. 2000, ApJ, 531, 861

Grady, C. A., Polomski, E. F., Henning, T., et al. 2001, AJ, 122, 3396

Harvey, P. M., Wilking, B. A., Joy, M., \& Lester, D. F. 1985, ApJ, 288, 725

Herbig, G. H. 1960, ApJS, 4, 337

Hony, S., Van Kerckhoven, C., Peeters, E., et al. 2001, A\&A, 370, 1030

Houk, N., \& Fuentes-Williams, T. H. 1982, in BAAS, 14, 615

Hudgins, D. M., \& Allamandola, L. J. 1999, ApJ, 516, L41

Jeffers, H. M., van Denbos, W. H., \& Greeby, F. M. 1963, Index catalogue of visual double stars, 1961.0 (Publications of the Lick Observatory, Mount Hamilton: University of California, Lick Observatory)

Keller, L. D., Sloan, G. C., Forrest, W. J., et al. 2008, ApJ, 684, 411

Kessler, M. F., Steinz, J. A., Anderegg, M. E., et al. 1996, A\&A, 315, L27 Lagage, P. O., Pel, J. W., Authier, M., et al. 2004, The Messenger, 117, 12

Lebouteiller, V., Bernard-Salas, J., Brandl, B., et al. 2008, ApJ, 680, 398

Leger, A., \& Puget, J. L. 1984, A\&A, 137, L5

Marraco, H. G., \& Rydgren, A. E. 1981, AJ, 86, 62

Meeus, G., Waters, L. B. F. M., Bouwman, J., et al. 2001, A\&A, 365, 476

Peeters, E., Hony, S., Van Kerckhoven, C., et al. 2002a, A\&A, 390, 1089

Peeters, E., Martín-Hernández, N. L., Damour, F., et al. 2002b, A\&A, 381, 571
Peeters, E., van Diedenhoven, B., Van Kerckhoven, C., et al. 2003, in Astrophysics of Dust

Persi, P., \& Tapia, M. 2003, A\&A, 406, 149

Povich, M. S., Stone, J. M., Churchwell, E., et al. 2007, ApJ, 660, 346

Racine, R., \& van den Bergh, S. 1970, in The Spiral Structure of our Galaxy, ed. W. Becker, \& G. I. Kontopoulos, IAU Symp., 38, 219

Reimann, H.-G., Linz, H., Wagner, R., et al. 2000, in Optical and IR Telescope Instrumentation and Detectors, ed. M. Iye, \& A. F. Moorwood, Proc. SPIE, 4008, 1132

Reipurth, B., Jewitt, D., \& Keil, K. 2007, Protostars and Planets V

Shore, S. N., Brown, D. N., Bopp, B. W., et al. 1990, ApJS, 73, 461

Siebenmorgen, R., Prusti, T., Natta, A., \& Müller, T. G. 2000, A\&A, 361, 258

Sloan, G. C., Jura, M., Duley, W. W., et al. 2007, ApJ, 664, 1144

Tielens, A. G. G. M. 2008, ARA\&A, 45, 289

Turon, C., Creze, M., Egret, D., et al. 1993, Bulletin d'Information du Centre de Données Stellaires, 43,5

van Boekel, R., Min, M., Waters, L. B. F. M., et al. 2005, A\&A, 437, 189

van den Ancker, M. E., Bouwman, J., Wesselius, P. R., et al. 2000, A\&A, 357, 325

van Diedenhoven, B., Peeters, E., Van Kerckhoven, C., et al. 2004, ApJ, 611, 928

Van Kerckhoven, C. 2002, Ph.D. Thesis, Instituut voor Sterrenkunde, Katholieke universiteit Leuven

Waelkens, C., \& Waters, L. B. F. M. 1997, in Infrared Space Interferometry: Astrophysics \& the Study of Earth-like Planets, ed. C. Eiroa, et al., 119

Waelkens, C., Waters, L. B. F. M., de Graauw, M. S., et al. 1996, A\&A, 315, L245

Waters, L. B. F. M., \& Waelkens, C. 1998, ARA\&A, 36, 233 